Zare-Behtash, H., Kontis, K., and Gongora-Orozco, N. (2008) Experimental investigations of compressible vortex loops. Physics of Fluids, 20 (12). p. 126105. ISSN 1070-6631

Copyright (C) 2008 American Institute of Physics

A copy can be downloaded for personal non-commercial research or study, without prior permission or charge

Content must not be changed in any way or reproduced in any format or medium without the formal permission of the copyright holder(s)

When referring to this work, full bibliographic details must be given

http://eprints.gla.ac.uk/83512

Deposited on: 18 June 2014

Enlighten - Research publications by members of the University of Glasgow http://eprints.gla.ac.uk 


\title{
Experimental Investigations of Compressible Vortex Loops
}

\author{
H. Zare-Behtash, ${ }^{*}$ K. Kontis, ${ }^{\dagger}$ and N. Gongora-Orozco ${ }^{\ddagger}$ \\ School of MACE, The University of Manchester, M60 1QD, UK
}

\begin{abstract}
The present study involves the shock wave and consequent vortex loop generated when a shock tube with various nozzle geometries is employed. It aims to provide quantitative and qualitative insight into the physics of these compressible phenomena. The geometries included two elliptic nozzles with minor to major axis ratios of 0.4 and 0.6 , a $15 \mathrm{~mm}$ circular nozzle and a $30 \times 30 \mathrm{~mm}$ square nozzle. The experiments were performed for driver gas (air) pressures of 4, 8 and 12 bar. Schlieren, shadowgraphy, and PIV techniques were employed to visualise and quantify the induced flow field.
\end{abstract}

\footnotetext{
*Researcher, The University of Manchester, UK, e-mail: h.zare-behtash@postgrad.manchester.ac.uk.

${ }^{\dagger}$ Reader, The University of Manchester, UK, e-mail: k.kontis@manchester.ac.uk.

${ }^{\ddagger}$ Researcher, The University of Manchester, UK, e-mail: n.gongora@postgrad.manchester.ac.uk.
} 


\section{INTRODUCTION}

Supersonic free jets are found in many applications involving jet and rocket propulsion, thrust vectoring, fuel injectors for supersonic combustion, gas stream emanating from the blast of a gun etc. Studies have shown that the major flow structure of supersonic jets is determined by nozzle pressure ratio and geometry. ${ }^{1,2}$ Non-circular jets provide efficient passive flow control at relatively low cost since they rely solely on changes in the geometry of the nozzle. The applications include improved large and small scale mixing, enhanced combustor performance, noise suppression, heat transfer, and thrust vector control (TVC). ${ }^{3}$ Non-circular injectors such as elliptic, triangular, and square nozzles are used to improve combustion processes by augmenting heat release, reducing emissions, and improving flame stability. Investigation into the properties of non-circular jets has been motivated by their enhanced characteristic entrainment properties relative to those of comparable circular jets. ${ }^{4}$ This enhanced entrainment is believed to be mainly the result of self-induced vortex ring deformations.

Due to the sensitivity of flows with concentrated vorticity to insertion of any probes, it is difficult to obtain accurate global measurements. Questions regarding the strength and location of vorticity in a particular flow need to be investigated. Vorticity is often compact, even though the velocity and pressure fields extend everywhere, and remains so by virtue of the Helmholtz laws of vortex motion, apart from viscous diffusion. ${ }^{5}$ A great deal of research is still ongoing to understand the physics of incompressible turbulence, and the presence of large property gradients characteristic of compressible flows exacerbates the complexity of the situation.

Detonations are distinguished from shock waves by the presence of an intrinsic length scale associated with a reaction zone. ${ }^{6}$ The study into the evolution of detonation waves that suddenly expand has been motivated not only by the need to suppress accidental detonations but also in the interest of the applicability of such flows to the concept of pulse detonation engines (PDEs). ${ }^{7-12}$ Upon diffraction of the detonation wave from a PDE, a vortex loop is formed immediately behind it.

Vortex loops exemplify the whole range of problems of vortex motion, and are relatively easy to produce. Once created, vortex loops are self-contained, auto motive and quite longevous. ${ }^{13}$ Their formation is a problem of vortex sheet dynamics, the steady state is a 
problem of existence, their duration is a problem of stability, and if there are several we have the problem of vortex interactions.

No matter the initial conditions in which a vortex loop is generated, there are three stages in the propagation of a vortex loop: formation, development and decay. Although numerous authors have studied both experimentally and numerically the formation, propagation and decay of incompressible vortex rings, ${ }^{14-20}$ relatively speaking, not much work covering the compressible ${ }^{21,22}$ and detonation ${ }^{23}$ regimes have been reported. Some numerical simulations assume an incompressible flow, while others take into account the effects of compressibility. ${ }^{24-26}$ What almost all of the numerical studies have in common is the assumption of axisymmetric flow, hence minimising computational time and requirements.

The following cold-flow study examines the diffracted shock wave pattern and the resulting vortex loop emitted from a shock tube of various exit geometries, and aims to provide a baseline for the future incorporation of an ejector at the exit of the tube. Three diaphragm pressure ratios of $P_{4} / P_{1}=4,8$, and 12 have been investigated.

Qualitative (schlieren and shadowgraphy) and quantitative (PIV) techniques have been utilised to elucidate the characteristic behaviour associated with compressible vortex loops of various shapes; such as the phenomenon of axis switching, instability vortices within the trailing shear layer due to Kelvin-Helmholtz (K-H) instabilities, the formation of counterrotating vortex loops ahead of the main one, the presence of an embedded shock wave within the main vortex loop, and finally the azimuthal instabilities which are believed to be the signal for the onset of vortex breakdown.

\section{EXPERIMENTAL SETUP}

\section{A. Shock Tube}

Experiments have been carried out using air as both the driver and driven gas with diaphragm pressure ratios $P_{4} / P_{1}=4,8$ and 12 . With $P_{4}$ being the pressure within the driving compartment of the shock tube, and $P_{1}$ the pressure inside the driven section.

An industrial film diaphragm divides the two sections of the shock tube. The thickness of the diaphragm was chosen to be 23,55 and $75 \mu \mathrm{m}$ for $P_{4} / P_{1}=4,8$ and 12 respectively. The diaphragm thicknesses corresponding to each pressure ratio were identical to those 
used by Kontis et al. ${ }^{27,28}$ They were chosen for being the minimum required to sustain the desired pressure without spontaneously rupturing them. The bursting of the diaphragm was initiated manually with a plunger. The tube was flushed with air after each run to remove any pieces of burst diaphragm.

Various adaptors were designed that could be attached to the end of the circular shock tube section (baseline) with i.d. $30 \mathrm{~mm}$ and o.d. $38 \mathrm{~mm}$. This allowed vortex loops of different shapes to be studied. They included two elliptical nozzles with minor to major axis ratios $(a / b)$ of 0.4 and 0.6 with $b=30 \mathrm{~mm}$, a circular nozzle with an internal diameter of $15 \mathrm{~mm}$, and a square nozzle with side lengths of $30 \mathrm{~mm}$. All nozzles had a wall thickness of $4 \mathrm{~mm}$, with a gradual area change from the $30 \mathrm{~mm}$ i.d. circular shock tube section (baseline) to the various shapes under consideration over a length of $300 \mathrm{~mm}$. The length of the circular driven section was $1310.5 \mathrm{~mm}$. The critical length of the driver section for the baseline section of the shock tube was $12.3 d_{i}, 8.53 d_{i}$, and $7.23 d_{i}$ for $P_{4} / P_{1}=4$, 8 , and 12 , respectively. This produces a pulsed upstream condition where the duration and magnitude of the pulse can be controlled up to the nozzles' inlet. ${ }^{28-30}$ The nozzle inlet conditions are not known and the flow conditions at the exit of the nozzles in terms of pressure and velocity vary in time. These will change for a fixed driver pressure when different nozzle geometries are used both because of different mass flows, inlet pressures and the transverse waves set up.

\section{B. Schlieren and Shadowgraphy}

High-speed schlieren and shadowgraph photography ${ }^{31,32}$ were employed to visualise the flow. The schlieren setup was identical to that used by An et al. ${ }^{33}$ and is shown schematically in Fig. 1. A transducer placed $660 \mathrm{~mm}$ from the diaphragm along the driven section of the shock tube (labelled T1 in Fig. 1), was used to trigger the flash lamp and to capture a single image. The pressure pulse generated by the passage of the shock wave acted as the trigger. Using an external delay generator the triggering of the flash lamp could be delayed and a sequence of images was captured. The exit times of the shock front from the different nozzles were different for a given incident shock and driver pressure due to changes in nozzle geometry. 


\section{Particle Image Velocimetry}

Particle Image Velocimetry is based on the measurement of the velocity of tracer particles carried by the fluid. ${ }^{34}$ For the current study, a high frame rate (1500 frames per second at $1024 \times 1024$ pixels resolution) PIV system consisting of a high repetition rate laser $(10 \mathrm{~mJ}$ at $1 \mathrm{kHz}$ ) for illumination, with a light arm for easy manipulation of the laser beam and a range of light sheet optics was used. A high frame rate camera (Photron APX RS) was employed. The data were recorded in an on-board memory of 8GB. A model 9306A TSI six-jet atomizer was used to generate oil droplets as small as $1 \mu \mathrm{m}$ in diameter. ${ }^{35}$ The particle relaxation time $\mathrm{e}^{36}$ for a $1 \mu \mathrm{m}$ olive oil particle was calculated as $\tau=2.2 \mu \mathrm{s}$.

A synchroniser allows control of timing of capture and has the facility for external inputs for triggering the PIV system. A high specification PC with TSI's Insight PIV software installed enables data download and analysis. In addition, TecPlot 10 is also loaded for data display and analysis (with TSI Plot PIV add-on).

The transducer placed along the shock tube (labelled T1 in Fig. 1) was used to generate an external trigger with different delays to capture frames at different intervals. The size of the interrogation zones $(32 \times 32)$ and the timing between the two PIV frames $(\Delta T=3 \mu s)$ was chosen based on the theoretical Mach number of the flow behind the incident shock wave $\mathrm{e}^{37}$ and the schlieren and shadowgraphs of the flow which were obtained prior to the PIV experiments. The total interrogation area was approximately $80 \mathrm{~mm} \times 90 \mathrm{~mm}$.

An enclosure was designed which encased the exit of the shock tube. Prior to each run, this enclosure was filled with tracer particles along with the driven section of the shock tube. The PIV setup is shown schematically in Fig. 2.

\section{RESULTS AND DISCUSSIONS}

\section{A. Experimental Uncertainty}

Uncertainty estimation given by Holman ${ }^{38}$ has been used to determine the uncertainties, in the form of error bars, in plotting the various vortex loop properties, such as nondimensional vortex loop diameter and distance travelled. The error analysis takes into consideration the resolution of the system, and the error due to the image processing.

The repeatability of the experiments is determined by: (i) setting the same driver pres- 
sure, (ii) having the same delay time output from the delay generator, and (iii) triggering the laser and camera at two consecutive frames separated by $\Delta T$. The aforementioned procedure was repeated twice and the location of the vortex loop for the two cases was compared frame by frame. The maximum difference between the location of the vortex loop for the sets of repeats was calculated as $1.5 \%$.

\section{B. Flow Properties}

Once the expansion wave from the end of the driver arrives at the nozzle exit it will reflect differently from the different nozzles and thus will superimpose different efflux conditions. The experimental Mach number of the shock at the exit $M_{s e}$, for the various driver pressures and nozzles used, is calculated from the PIV velocity data as the shock exits the tube. Table I gives the experimental conditions for the different test cases.

As the incident shock travels along a converging nozzle, such as the circular one, it is strengthened by the area reduction. ${ }^{39-42}$ For the square nozzle, however, since the area is increased, the strength of the incident shock is reduced. Chisnell ${ }^{39}$ derived a first order relationship between changes in area and shock strength for a shock moving through a small area change. Given the value of the shock strength $(z)$ on encountering an area change in a channel and the ratio of the areas at the ends of the variable area section, the strength of the emerging shock can be obtained. For the present cases, it has been calculated and the theoretical Mach numbers are presented in Table I. The differences between the experimental and theoretical Mach numbers are also given in the same table.

Discrepancies between the theoretical and experimental results are attributed to the disturbances generated by the motion of the incident shock, especially at higher Mach numbers (the circular nozzle case) where the effects of these disturbances are more pronounced due to higher pressure ratios. Experiments performed by Bird. ${ }^{42}$ showed that with a gradual area change (similar to the present study) the experimental value of the shock strength is much closer to the theoretical ones. In a situation where the strength varies rapidly along the shock front, this bears little relevance to the true unsteady shock strength at a given point on the front. The average value approximates to the unsteady strength only when applied to a portion of the shock front across which the strength is not changing rapidly. ${ }^{43}$

The $R e$ number, $R e=\rho u_{p} L / \mu$, was calculated in terms of the density behind the incident 
shock $\rho$, the induced velocity behind the incident shock $u_{p}$ obtained from PIV measurements, and the dynamic viscosity $(\mu)$ corresponding to the flow behind the incident shock. The length scale $(L)$ used in the calculation of $R e$ is dependent on the shape of the nozzle employed: for the square nozzle $L=30 \mathrm{~mm}$, for the circular nozzle $L=15 \mathrm{~mm}$, and for the elliptic nozzles $L=12 \mathrm{~mm}$ and $L=18 \mathrm{~mm}$ for $a / b=0.4$ and 0.6 respectively.

\section{Effect of Nozzle Geometry}

1. Elliptic Nozzle $a / b=0.4$

Figure 3 shows a schematic of the main features of a diffracted shock wave. It presents the plane incident wave, the diffracted wave, and the front of the reflected expansion wave (sound wave) which propagates back into the post-shock region and is the demarcation between uniform and non-uniform flow. ${ }^{44,45}$ With increasing Mach number the reflected sound wave does not propagate upstream as rapidly as in the lower Mach number case because of the greater velocity of the oncoming gas. The expansion of the flow at the corner accelerates the gas, at the same time turning it parallel to the slipstream. ${ }^{46,47}$ The slipstream is due to the separation of the gas and is brought about by the inability of the flow to negotiate the corner.

Except for small corner angles $\left(15^{\circ}, 30^{\circ}\right.$ and $\left.45^{\circ}\right)$, the overall pattern remains qualitatively the same for any given shock Mach number. Numerical simulations of Sun and Takayama ${ }^{48}$ revealed that: the vorticity production increases sharply when the corner angle is varied from $15^{\circ}$ to $45^{\circ}$. However, for corner angles over $90^{\circ}$, the rate of vorticity production hardly increases and reaches to a constant value.

Figure 4 presents the oblique views of the flow corresponding to the vortex loop formation and propagation from the elliptical nozzle with minor to major axis ratio of 0.4 . The times are given from the instant the incident shock exits the tube. The elliptic shape of the shock wave and accompanying vortex loop is best viewed in Fig. 4(a). The vortex loop generated from shock diffraction is due to baroclinic effects. ${ }^{49}$

Unlike conventional circular vortex loops, when a change in radius of curvature is introduced to the nozzle generating the vortex loop, it leads to a non-uniform translational velocity downstream. From Fig. 4, the portion of the loop that is generated from the corners 
has a greater velocity downstream of the nozzle leaving the upper and lower parts of the vortex loop behind. This phenomenon results from self-induced Biot-Savart deformation ${ }^{3}$ of vortex loops with non-uniform azimuthal curvatures. The parts which are travelling slower downstream are in fact travelling outwards tending to stretch the vortex loop in the vertical direction (Fig. 4(b)). In the same figure we notice the presence of a vortex pair just behind the primary vortex loop.

Observing Figs. 5(a) and 5(b), which show the vortex loop behaviour at later times, we begin to see small instability vortices to form. This observation has also been made on circular vortex rings. ${ }^{30}$ At the same time that these vortices begin to form in the shear layer we notice the distortion of the vortex loop from its near perfect shape. These instabilities grow with time as seen in Fig. 5(b). In Fig. 5(b) there appears to be a smaller vortex loop just ahead of the primary one. The direction of circulation is similar to the primary loop, indicated by the bright and dark colours of the cores. This is the same vortex pair that was initially identified in Fig. 4(b). The generation of the vortex is attributed to the occurrence of a velocity peak at the nozzle exit. ${ }^{13}$

Results of the PIV experiments performed at $P_{4} / P_{1}=4$ on the 0.4 elliptic nozzle are shown in Fig. 6. The colours present the vorticity profile calculated as $\omega_{z}=\frac{\partial u}{\partial y}-\frac{\partial v}{\partial x}$, while the vectors show the velocity magnitude $\left(\sqrt{U^{2}+V^{2}}\right)$, where the magnitude of the velocity is proportional to the length of the vectors. Similar to the schlieren and shadowgraphs, $t=0$ is the time the shock exits the tube. The $x$-axis is measured by the software from the edge of the raw PIV image recorded by the camera, and the interrogation zones were chosen to start $\approx 5 \mathrm{~mm}$ from the nozzle exit to avoid any reflections from the nozzle interfering with the signal from the tracer particles.

In Fig. 6(a) we can see the presence of the incident shock wave depicted by the vectors and also the emergence of the vortex loop. We can immediately conclude that the maximum velocities occur within the region of the vortex loop and also just in front of it. This is because at these locations the flow is accelerated due to the combined circulation of the upper and lower cores. As the vorticity of the main cores continues to grow so does the strength of the vortices within the shear layer, suggesting a relationship between the strength of the primary vortex loop and that of the shear layer instability vortices. During the laminar phase, the core structure is highly concentrated with peak vorticity values as shown in Fig. 6(b). Azimuthal bending instabilities mark the beginning of the transition stage; the upper 
and lower cores become deformed and show signs of the turbulent breakup (Fig. 6(c)). The turbulent stage is characterised by a strong shedding process into the wake of the vortex loop, indicated by the formation of small and concentrated vorticity regions in the periphery of the core regions. However, the vorticity distribution in the core region remains concentrated. ${ }^{50}$

Figure 7 is a visualisation of the flow pattern at a driver pressure of 8 bar at three different times. The first column (Figs. 7(a), 7(c) and 7(e)) represents shadowgraphs of the flow whilst the second column (Figs. $7(\mathrm{~b}), 7(\mathrm{~d})$ and $7(\mathrm{f})$ ) are images of the same flow but with the nozzle rotated through $90^{\circ}$ so that we are looking along the minor axis. The shock cells are larger compared to the lower driver pressure case due to the higher flow Mach number. The axial flow velocity in the recirculating region of the vortex loop becomes supersonic in the frame of reference of the vortex loop, producing a rearward-facing shock wave embedded in this region.

In Fig. 7(a) we observe the formation of the diamond shock structure behind the vortex loop. These are formed by the reflection of the expansion fan at the jet boundary as a compression wave. The compression waves coalesce into shock waves undergoing either regular or Mach reflection depending on the underexpansion of the jet. ${ }^{51} \mathrm{~A}$ small flattening of the vortex loop apex is also apparent in the aforementioned figure due to the deceleration of the flow through the diaphragm shock. The embedded or diaphragm shock is best visible in Fig. 7(b) with the nozzle rotated. For non-axisymmetric jets the level of underexpansion can be different for the same jet when viewed from different directions due to the complex jet geometry and the aspect ratio. ${ }^{52}$

At later times (Figs. 7(c) and 7(e)) the vortex loop increases in size while being stretched in the vertical direction. Just after the central portion of the diaphragm shock, the flow is divided into two regions: (i) the flow along the central portion generated due to the presence of the rear-ward facing diaphragm shock; and (ii) the flow above and below the central axis divided by two slip streams in Fig. 7(c). The slipstream is generated in order to accommodate the turning motion of the flow passing through the diaphragm shock and drawn into the circulation of the cores. ${ }^{53}$ The rotated image of Fig. $7(\mathrm{~d})$ shows the flow becoming more turbulent between the primary vortex loop and the jet flow behind it. This is indicated by the increase in the small vortices visible in the shadowgraph.

What it can be discerned from Figs. $7(\mathrm{e})$ and $7(\mathrm{f})$ is that the vortex loop does not expand along the major axis as much as along the minor one due to the phenomenon of axis 
switching.

Analysis of the PIV images shown in Fig. 8, which correspond to the same flow conditions as in Fig. 7, indicates the strong centripetal force present in the core of the vortex loop causing the particles within the core to be thrown out, especially at later times.

At high Reynolds numbers, vortex loops become even more unstable and shed significantly more vorticity into the wake. The wake of the vortex loop is instability free in the initial phases of propagation (Fig. 8(a)). In Fig. 8(b) the vorticity field begins to stretch, whilst the magnitude of vorticity remains roughly the same. A new region is beginning to form ahead of the primary vortex loop with the same direction of vorticity. Since the laser sheet is cutting through the central axis of the nozzle, this new vorticity region is the jet stream which is going through the centre of the vortex loop. This is visible in the vorticity contours shown in Figs. 8(b) and 8(c). As the primary vortex loop continues to grow in diameter it sheds vorticity into its wake (Fig. $8(\mathrm{~d})$ ) as it leaves the field of view. This result was also obtained in the direct numerical simulations (DNS) of viscous, laminar vortex rings by James and Madnia. ${ }^{54}$ Eventually, the vortex loop motion ceases as all of its vorticity is deposited into the wake and is spread by viscous diffusion. ${ }^{55}$

With increasing driver pressure, the vortex loop development is accompanied by the generation of intense disturbances. ${ }^{56}$ This can be seen in Fig. 9. This is due to the interaction between the diaphragm shock present within the core of the vortex loop. Strong interactions involve significant deformation of the shock wave due to the vortex and include the production of secondary shocks due to the phenomenon of shock splitting. ${ }^{57,58}$ The edges of the initially planar diaphragm shock are drawn into the cores of the vortex and diffract around it. This leads to the generation of compression waves around the periphery of the primary loop. The vorticity profile for the extremum of pressure setting is qualitatively similar to that of the intermediate pressure presented in Fig. 8.

With increasing driver pressure and hence Mach number, the slipstream increases its angle relative to the horizontal. This causes the diameter of the vortex loop to be innately larger at higher Mach numbers, as shown in Fig. 10(a). The diameter of the vortex loop, $D_{r}$, is non-dimensionalised with reference to the minor axis of the nozzle, $d_{i}$. The dimensionless time $\left(t-t_{1}\right) \times U_{s} / d_{i}$ is based on the specified time $t$, the time of the initial emergence of the vortex loop $t_{1}$, the corresponding shock wave velocity $U_{s}$, and the minor axis of the nozzle $d_{i}$. At the lowest driver pressure $(4$ bar $)$, the diameter of the vortex loop grows to 
approximately four times the height of the minor axis; afterwards it seems to remain almost uniform. However, further studies are required to justify the latter statement. This value is 4.5 for the intermediate driver pressure. Following the same trend, one would expect the vortex loop with the extreme driving pressure to behave in a similar fashion, however, sufficient data is not available to confirm this. The rate the vortex loop travels, shown in Fig. 10(b), does not appear to be affected by the variation in flow Mach number. The distance propagated by the vortex loop was measured from the exit of the nozzle until the location where the vortex core could be identified in the flow.

\section{Elliptic Nozzle $a / b=0.6$}

Figure 11 shows the oblique view of the vortex loop when using an elliptical nozzle with a minor to major axes ratio of 0.6 and $P_{4} / P_{1}=4$. In comparison to the vortex loop generated from the smaller elliptical nozzle, the different downstream translational velocity of the various parts of the larger vortex loop are not as apparent. This is because with an axis ratio of 0.6 , the nozzle is closer to the conventional axisymmetric circular vortex ring in which all parts of the ring travel downstream with the same velocity. The lack of supersonic concentric flow precludes the formation of the diaphragm shock at this stage. The time scale of the rolling up of the vortex sheet is larger at lower driver pressures. This leads to a thicker vortex core. The expansion of the core diameter leads to more stable vortex loops. ${ }^{59,60}$

Observing the PIV results for this particular nozzle, shown in Fig. 12, we note the reduced magnitude of vorticity in comparison to the smaller elliptic vortex loop case (Fig. 6). The region of the entrained ambient fluid for the larger elliptical vortex loop is much greater compared the smaller vortex loop. The vorticity continues to grow until it reaches a maximum at the point known as pinch-off where the vortex loop separates from the flow exiting the tube which used to feed energy into the vortex loop. ${ }^{30}$ According to Shusser and Gharib: ${ }^{19}$ at the vortex pinch-off the translational velocity of the ring equals to the jet flow velocity near the vortex loop. This location, is indicated by the increase in vorticity and also the reduction in the flow velocity between the main vortex cores and the exit of the tube (Fig. 12(b)).

Kelvin-Helmholtz (K-H) shear layer instabilities on the gas front of the jet generate vortices behind the primary vortex loop. ${ }^{24}$ The development of these instabilities on the 
shear layer generally results in an S-shaped pattern containing pair of vortices of the same sign. K-H instability development is due to the presence of a velocity jump at the interface between the flows exiting the tube and the ambient. $\mathrm{K}-\mathrm{H}$ instabilities are minimised when the velocity of the inner and outer jets are similar. ${ }^{61}$ This can be seen when comparing Figs. 12 and 13.

Figure 14 shows the flow characteristics at three different times using the maximum driver pressure for the current nozzle $\left(P_{4}=12\right.$ bar $)$. In Fig. 14(a) we can observe the diffracted incident shock wave along with the vortex loop with the diaphragm shock wave. The diaphragm shock is carried along at the same speed as the vortex loop. ${ }^{62}$ It is a rearward facing shock such that there is low pressure to the left of the shock and high pressure to the right. The diaphragm shock forms because the subsonic flow is accelerated to sonic velocity as it expands around the corner and the sonic line is then followed by a PrandtlMeyer expansion and then by the upward facing shock necessary to balance the pressures. According to Sun and Takayama ${ }^{63}$ the smallest incident shock Mach number $M_{s}$ at which a secondary shock wave starts to appear is $M_{s}=1.34$. In the same figure we notice the formation of a small cone shaped nozzle along the central axis of the vortex loop. This is due to the Mach reflection of the shock waves within the jet. The shadowgraph of the flow with the nozzle rotated $90^{\circ}$ shows the affect of nozzle aspect ratio on the behaviour of the flow (Fig. 14(b)).

At a later time (Figs. 14(c) and 14(d)), the deceleration of the flow caused by the generation of a counter-rotating vortex loop ahead of the primary one becomes intensified. The jet boundary deforms by contracting upstream of the vortex and expanding downstream, antisymmetrically about the vortex plane (Fig. 14(c)). This deformation of the jet boundary results in a transfer of momentum from the jet to the vortex ring, so that its radius gradually increases. In Fig. 14(c) we see compression waves emanating from the two vortex cores as result of the interaction between the vortex and the now slightly curved diaphragm shock. These waves are, however, not visible in the rotated nozzle case due to the three dimensional nature of the flow.

As the vortex loop continues to grow due to entrainment and viscous effects, it becomes quite bulky in shape (Fig. 14(e)). The diaphragm shock visible in Fig. 14(f) slows down the flow across it and creates an indent in the apex. Due to nozzle geometry and the local interaction between waves in the flowfield,${ }^{64}$ the shock patterns in the trailing jet are different 
in Figs. 14(e) and 14(f).

The location of the incident shock as well as the formation of the vortex loop is identifiable in PIV results presented in Figs. 15(a) and 15(b). The vortex loop expands immediately after its generation, causing a fast increase in vortex loop diameter. The counter-rotating vortex loop ahead of the primary one is also visible in the modified vorticity contour of Fig. 15(c). As the vorticity diffuses out of the body of the moving vortex loop into the outer irrotational fluid, it has two effects: (i) it causes some of the fluid, with newly acquired vorticity, to be entrained into the interior of the bubble (Fig. 14(e)), while (ii) the rest is left behind and accounts for the appearance of vorticity in a wake (Fig. 15(d)). ${ }^{50,55}$

The maximum non-dimensional diameter of the 0.6 elliptic vortex loop is relatively smaller than the previously discussed 0.4 vortex loop case (see Fig. 16(a)). The difference between the vortex loop diameters at different driver pressures is more pronounced. The general behaviour is that the vortex loop diameter continues to grow until approximately 7 time steps, where it reaches a uniform size. The rate of propagation of the vortex loop is quite linear with time, it is not affected by the change of vorticity strength along its path.

\section{Circular Nozzle}

The shadowgraphs revealing the motion of the rather small circular vortex ring are presented in Fig. 17. Unlike the vortex loops presented so far, in this particular case the entire ring has the same translational velocity in the initial stages of propagation. The adverse pressure gradient due to the diaphragm shock causes the flow to decelerate more near the central region compared to the edge of the embedded shock which results in a strong velocity gradient. Thus, a shear layer is formed ahead of the ring which rolls up due to Kelvin-Helmholtz instability into a counter rotating vortex ring (Fig. 17(a)). The circulation of the counter rotating vortex ring is opposite to that of the primary ring and hence, the self induced velocity of it is towards the upstream direction. It rolls over the periphery of the primary loop (Figs. 17(b) and 17(c)) and moves in the upstream direction with respect to the primary ring. Eventually it is ejected from the primary ring, continues to move in the upstream direction relative to the primary ring and interacts with the trailing jet (Fig. 17(d)). In Fig. 17(b) the segment containing the Mach reflection along with the consequence slip line and triple point have been enlarged in the figure. 
The PIV results indicate that as the vortex ring is generated and travels downstream (Fig. 18(a)), the circular shape of the core begins to deform. This is depicted in the vorticity contours of Figs. 18(b) and 18(c). This is due to the generation of the secondary vortex loop ahead of the primary one described in Fig. 17, which decelerates the flow at the apex of the primary loop leading to the flattening of the vorticity contours visible in Fig. 18(c). As the secondary vortex loop passes over the periphery of the primary one and into its wake, the cores are stretched downstream and the ring loses its symmetry while the local vorticity intensifies as it is shown in Fig. 18(d). This behaviour is similar to the numerical findings by Archer et al. ${ }^{65}$ The shear layer instabilities fed into primary loop, increase in magnitude and irregularity leading to a very random motion within the shear layer. As vorticity is dumped into the wake in Fig. 18(e), its magnitude within the primary vortex loop begins to decrease gradually (Fig. 18(f)). The counter-rotating vortex loop which has now passed over the periphery of the primary vortex loop can be identified in the modified vorticity field of Fig. 18(f).

In the oblique schlieren view presented in Fig. 19(a), the vortex loop along with the shock interactions can be seen. The secondary counter-rotating vortex loop is also apparent. Through Figs. 19(b)-19(c) we notice the growth of the secondary vortex loop together with the formation of a Mach disk. In Fig. 19(c), the diameter of the counter-rotating vortex has grown larger than the primary loop as it begins to pass over it.

Although for lower driver pressures the vortex core is nearly circular, for higher pressures, however, the vortex cores are spread due to the higher stream-wise velocity. The vorticity contours of Fig. 20 show how the vortex cores are bent outwards as the counter-rotating vortex moves around it (Figs. 20(a)-20(d)). The magnitude of the vorticity of the primary loop changes as it interacts with the secondary one tending to decrease. This is because once the counter-rotating vortex loop is aligned vertically with the primary loop, the circulation of the secondary vortex loop reduces the vorticity magnitude of the primary one, because they are acting in opposite directions. After the secondary loop has passed completely into the wake of the primary one, the vorticity of the primary vortex loop increases (Fig. 20(e)) until it becomes turbulent and begins to shed significant amounts of vorticity (Fig. 20(f)). The increased amount of streamwise vorticity within the wake signals the beginning of vortex loop decay. The study of Bergdorf et al. ${ }^{66}$ revealed that the streamwise vorticity consists of axial structures shed along the wake of the vortex loop and large-scale vortex structures 
that are still attached to the inside of the vortex loop.

The change in vortex loop properties with time are given in Fig. 21. For all driver pressures investigated, the rate of vortex loop growth varies linearly with time but begins to level after a distance equal to $2 d_{i}, 2.5 d_{i}$, and $3.25 d_{i}$ in order of increasing driver pressure, where $d_{i}$ is the internal diameter of the tube. The rates of propagation for the two higher driver pressures where we have the presence of the diaphragm shock are similar. It is conjectured that the presence of the diaphragm shock makes independent the initial flow conditions; at a lower driver pressure the strength of the shock is smaller as are its effects, for a higher driver pressure the effects of the diaphragm shock are stronger. Hence, the effects are relative, and because the diaphragm shock is moving with the vortex loop it influences the rate of propagation.

\section{Square Nozzle}

The vortex loop shown in Fig. 22 is initially square and coplanar. The induced velocity is highest in regions of the highest curvature. ${ }^{67}$ This is due to the non-uniform flow expansion which is higher near the corners than the side walls. Following the three-dimensional motion of the loop carefully we observe that as the corner portions move ahead (Figs. 22(a) and 22(b)), the remaining side portions develop a higher curvature and with this high curvature they start moving with an increasing velocity until they arrive at the location of the corner portions (Figs. 22(c) and 22(d)). Along with this alignment comes the process of axes switching. After the loop has aligned, it again behaves in the same manner i.e., the sections which are aligned with the corner portions protrude forward, traveling faster than the rest of the loop. Again the regions left behind develop an increased curvature and velocity and the loop aligns once more. But this time because of the inherent azimuthal disturbances the alignment is not so perfect.

Axis switching is caused by the azimuthal non-uniformities. It results from a faster growth rate of the jet's shear layers in the direction of minor axis than that of the major axis. In a square jet, the minor and major axes are denoted as directions normal to the flat side, and along the diagonal direction, respectively.

Wang et al. ${ }^{61}$ observed four pairs of vortices (each located at the end of the major axis) occurring close to the nozzle at $x / D_{e}=0.28$, as shown in Fig. 23(a) (where $D_{e}$ is the 
equivalent diameter of the square nozzle). The fluid between the two vortex pairs is induced inwards (inflow pair). The inflow vortices result in a contraction of the jet cross-section in the direction of the major axis. With downstream distance, each vortex pair moves towards the jet centreline due to the mutual induction and, as a result, the two adjacent vortices on a side move closer to each other and form an outflow pair. Consequently, the cross-section rotates $45^{\circ}$ at $x / D_{e}=1.25$ (Fig. $23(\mathrm{~b})$ ).

The results of the PIV experiments performed on the square nozzle (Fig. 24) revealed that almost as soon the vortex loop is generated there appear instability vortices within the shear layer leading up to the vortex cores and also in the region between the two upper and lower cores (Figs. 24(b) and 24(c)). In the PIV images presented in Fig. 24 we can notice that the induced vorticity field of the two cores is stretched in the streamwise direction indicating that the circulation within the core is affecting mostly the fluid upstream and downstream of the core (horizontal direction), relative to the fluid in the transverse direction. The random motion between the different time frames is due to the irregular azimuthal disturbances which have spread over the entire loop, hence the portion (slice) of the loop, we are observing, exhibits a transient behaviour which gives rise to the different shapes and sizes of the high vorticity regions in the PIV results. As put nicely by Lugt:68 "instability is a mechanism by which a fluid accommodates to strong forces, and new flow patterns are created."

The behaviour of the square vortex loop for the highest driver pressure is shown in PIV images of Fig. 25. Unlike any of the shapes and driver pressures studied, for this particular case, the vorticity in the shear layer is quite large and stretches to about twice the height of the nozzle $(60 \mathrm{~mm})$ downstream. The shear layer is quite unstable and oscillates in space as it is visible in Figs. 25(c) and 25(d). The magnitude of the vorticity within the shear layer is higher than that of the vortex loop with a region of very uniform flow between the upper and lower shear layers.

The diameter of the vortex loop increases quite rapidly during the first segment of the vortex loop propagation for all driver pressures tested (Fig. 26(a)). The diameter then saturates and after a short period it again continues to increase. Up to the point where the diameter initially increases, the rate at which the vortex loop travels is independent of flow Mach number (Fig. 26(b)). As soon as the vortex loop diameter begins to level off (approximately 5 time steps), the rate of propagation of the vortex loop for the different 
driver pressures, begin to diverge, with the vortex loop formed by the lower driver pressure covering the least distance and the vortex loop generated by the higher driver pressure covering the most.

\section{Conclusions}

The present study involved the shock wave and consequent vortex loop generated when a shock tube with various nozzle geometries is employed. These include: two elliptical nozzles with minor to major axes ratios of 0.4 and 0.6 , a $15 \mathrm{~mm}$ diameter circular nozzle, and a $30 \times 30 \mathrm{~mm}$ square. The experiments were performed for driver gas (air) pressures of 4, 8, and 12 bar; with the pressure in the driven section being atmospheric. Using schlieren and shadowgraphy along with the PIV technique the formation and propagation of the various types of vortex loops generated were examined.

Although in most numerical simulations the flow is assumed to be axisymmetric the result of the current study suggest that even at the early phases of vortex loop propagation the flow is three-dimensional. Viscous diffusion of the momentum contained in the vortex loop core causes the diameter to grow, with ambient fluid being engulfed, or entrained, into the core. The total circulation then decays, thereby decreasing the velocity of propagation of the loop. This decaying process leads to eventual annihilation of all vorticity contained in a vortex.

At driver pressures $P_{4}=8$ and 12 bar, a rear-ward facing diaphragm or embedded shock forms in the core of the vortex loops. The shock wave results in the formation of a shear layer ahead of the primary vortex loop which rolls up due to Kelvin-Helmholtz instability into a counter rotating vortex.

The PIV analysis revealed that for some cases, for example, the elliptic vortex loop with axes ratio of 0.4 , the vorticity is somewhat focused around the main vortex cores while in other cases, such as the square vortex loop, the area which is influenced by the cores is stretched in the stream-wise direction.

For almost all the driver pressures and shapes studied, the rate of vortex loop propagation is independent of flow Mach number. This takes into account the presence of the diaphragm shock and also the counter-rotating vortex loop which is generated at certain flow conditions.

A feature which has been present in all the cases studied is the presence of the instability 
vortices in the wake of the main vortex loop. Although these tiny instabilities start off as very weak, they soon become stronger and more distributed within the region bounded by the upper and lower vortex cores. It is these random disturbances which are initially fed through the shear layer which eventually give rise to the azimuthal disturbances leading to the complete random, irregular, and chaotic motion of the flow.

Further PIV studies will be conducted to analyze the vortex loops generated by rotating the nozzles so that we are looking at the vortex loops from a different perspective. The new set of PIV measurements will also include the study of the flow when the light sheet is placed normal to the direction of travel so that we can capture the entire vortex loop as viewed from the front. By doing so we may be able to capture the vortex pairs at the corners which cause the phenomenon of axis switching.

\section{Acknowledgments}

The authors are indebted to the technical staff at The University of Manchester for their assistance, for the help and advice of Dr. Martin Hyde (TSI) for the installation and setup of the PIV system, and Mr. Parham Momeni for assistance in presentation of the manuscript. The support of the EPSRC Engineering Instrument Pool, for the loan of the PIV system as well as the financial support of the EPSRC are greatly acknowledged. We would also like to take the opportunity to thank the reviewers for their constructive comments. 
1 Love, E. S., Grigsby, C. E., Lee, L. P., Woodling, M. J., "Experimental and theoretical studies of axisymmetric free jets," NASA technical report, NASA TR-R-6, (1959).

${ }^{2}$ Kweon, Y. H., Miyazato, Y., Aoki, T., Kim, H. D., "Experimental investigation of nozzle exit reflector effect on supersonic jet," Shock Waves 15, pp. 229-239, (2006).

3 Gutmark, E.J., Grinstein, F.F., "Flow control with noncircular jets," Annual Review of Fluid Mechanics 31, pp. 239-272, (1999).

4 Grinstein, F. F., Gutmark, E., Parr, T., "Near field dynamics of subsonic free square jets. A computational and experimental study," Physics of Fluids 7, pp. 1483-1497, (1995).

5 Saffman, P. G., "Dynamics of vorticity," Journal of Fluid Mechanics 106, pp. 49-58, (1981).

6 Shepherd, J. E., Schultz, E., Akbar, R., "Detonation diffraction," In: Ball, G., Hillier, R., Roberts, G., (eds.) Proceedings of the 22nd International Symposium on Shock Waves 1, pp. 41-48, (2000).

7 Pantow, E.G., Fischer, M., Kratzel, T., "Decoupling and recoupling of detonation waves associated with sudden expansion," Shock Waves 6, pp. 131-137, (1996).

8 Papalexandris, M.V., Thomas, J.F., Jacobs, C., Deledicque, V., "Structural characteristics of detonation expansion from a small channel to a larger one," Proceedings of the Combustion Institute, pp. 2407-2414, (2007).

9 Ohyagi, S., Obara, T., Hoshi, S., Cai, P., Yoshihashi, T., "Diffraction and re-initiation of detonations behind a backward-facing step," Shock Waves 12, pp. 221-226, (2002).

10 Glaser, A.J., Caldwell, N., Gutmark, E., Hoke, J., Bradley, R., Schauer, F., "Effects of tube and ejector geometry on the performance of pulse detonation engine driven ejectors," 42nd AIAA/ASME/SAE/ASEE Joint Propulsion Conference 8 Exhibit, California, AIAA 20064790 (2006).

11 Eustace, V.A., "A study of two-dimensional supersonic air ejector systems," PhD Thesis, The University of Manchester Institute of Science and Technology, (1969).

12 Glaser, A.J., Caldwell, N., Gutmark, E., Hoke, J., Bradley, R., Schauer, F., "Experimental study of ejectors driven by a pulse detonation engine," 45th AIAA Aerospace Sciences Meeting and exhibit, Reno, AIAA 2007-447 (2007).

13 Dziedzic, M., Leutheusser, H. J., "An experimental study of viscous vortex rings," Experiments 
in Fluids 21, pp. 315-324, (1996).

14 Takamoto, M., Izumi, K., "Experimental observation of stable arrangement of vortex rings," Physics of Fluids 24, pp. 1582-1583, (1981).

15 Dhanak, M.R., DeBernardinis, B., "The evolution of an elliptic vortex ring," Journal of Fluid Mechanics 109, pp. 189-216, (1981).

16 Oshima, Y., Izutsu, N., Oshima, K., Hussain, A.K.M.F., "Bifurcation of an elliptic vortex ring," Fluid Dynamics Research 3, pp. 133-139, (1988).

17 Grinstein, F.F., DeVore, C.R., "Dynamics of coherent structures and transition to turbulence in free square jets," Physics of Fluids 8, pp. 1237-1251, (1996).

18 Yoon, J.H., Lee, S.J., "Investigation of the near-field structure of an elliptic jet using stereoscopic particle image velocimetry," Measurement Science and Technology 14, pp. 2034-2046, (2003).

19 Shusser, M., Gharib, M., "Energy and velocity of a forming vortex ring," Physics of Fluids 12, pp. 618-621, (2000).

20 Zhao, W., Frankel, S. H., Mongeau, L. G., "Effects of trailing jet instability on vortex ring formation," Physics of Fluids 12, pp. 589-596, (2000).

21 Jiang, Z., Onodera, O., Takayama, K., "Evolution of shock waves and the primary vortex loop discharged from a square cross-section tube," Shock Waves 9, pp. 1-10, (1999).

22 Minota, T., "Interaction of a shock wave with a high-speed vortex ring," Fluid Dynamics Research 12, pp. 335-342, (1993).

23 Bykovets, A. P., Repin, V. B, "Formation of a vortex rings at the open end of a pulsed chamber," Combustions, Explosions and Shock Waves 16, pp. 73-77, (1980).

24 Minota, T., Nishida, M., Lee, M. G., "Shock formation by compressible vortex ring impinging on a wall," Fluid Dynamics Research 21, pp. 139-157, (1997).

25 Takayama, F., Ishii, Y., Sakurai, A., Kambe, T., "Self-intensification in shock wave and vortex interaction," Fluid Dynamics Research 12, pp. 343-348, (1993).

26 Ebrahimi, H. B., Mohanraj, R., Merkle, C. L., "Multilevel analysis of pulsed detonation engines," Journal of Propulsion and Power 18, pp. 225-232, (2002).

${ }^{27}$ Kontis, K., An, R., Edwards, J.A., "Compressible vortex-ring interaction studies with a number of generic body configurations," AIAA Journal 44, pp. 2962-2978, (2006).

28 Kontis, K., An, R., Zare-Behtash, H., Kounadis, D., "Head-on collision of shock wave induced 
vortices with solid and perforated walls," Physics of Fluids 20, pp. 1-17, (2008).

29 Brouillette, M., Hebert, C., "Propagation and interaction of shock-generated vortices," Fluid Dynamics Research 21, pp. 159-169, (1997).

30 Arakeri, J.H., Das, D., Krothapalli, A., Lourenco, L., "Vortex ring formation at the open end of a shock tube: A particle image velocimetry study," Physics of Fluids 16, pp. 1008-1019, (2004).

31 Settles, G.S., "Schlieren and Shadowgraph techniques," Springer, (2001).

32 Merzkirch, W., "Flow Visualization," Academic Press Inc, (1974).

33 Kontis, K., Kounadis, D., An, R., Zare-Behtash, H., "Vortex Ring Interaction Studies with a Cylinder and a Sphere," International Journal of Heat and Fluid Flow 29, pp. 1380-1392, (2008).

34 Raffel, M., Willert, C.E., Kompenhans, J., "Particle Image Velocimetry," Springer, (1998).

35 Megerle, M., Sick, V., Reuss, D. L., "Measurements of digital particle image velocimetry precision using electro-optically created particle image displacements," Measurement Science and Technology 13, pp. 997-1005, (2002).

36 Melling, A., "Tracer particles and seeding for particle image velocimetry," Measurement Science and Technology 8, pp. 1406-1416, (1997).

37 Anderson, J.D., "Modern Compressible Flow, with Historical Perspective," McGraw-Hill, Inc, ed. 2, (1990).

38 Holman, J. P., "Experimental Methods for Engineers," McGraw-Hill, Inc, (1994).

39 Chisnell, R.F., "The motion of a shock wave in a channel, with applications to cylindrical and spherical shock waves," Journal of Fluid Mechanics 2, pp. 286-298, (1957).

40 Allgood, D., Gutmark, E., Rasheed, A., Dean, A.J., "Experimental investigation of a pulse detonation engine with a two-dimensional ejector," AIAA 43, pp. 390-398 (2005).

41 Russell, D.A., "Shock-wave strengthening by area convergence," Journal of Fluid Mechanics 27, pp. 305-314 (1967).

42 Bird, G.A., "The effect of wall shape on the degree of reinforcement of a shock wave moving into a converging channel," Journal of Fluid Mechanics Digital Archive 5, pp. 60-66, (1959).

43 Sloan, S.A., Nettleton, M.A., "A model for the decay of a wall shock in a large abrupt area change," Journal of Fluid Mechanics 88, pp. 259-272 (1978).

44 Hillier, R., "Computation of shock wave diffraction at a ninety degrees convex edge," Shock 
Waves 1, pp. 89-98, (1991).

45 Griffith, W., Brickl, D. E., "The diffraction of strong shock waves," Physical Review 89, pp. 451-453, (1953).

46 Skews, B. W., "The perturbed region behind a diffracting shock wave," Journal of Fluid Mechanics 29, pp. 705-719, (1967).

47 Skews, B. W., "The shape of a diffracting shock wave," Journal of Fluid Mechanics 29, pp. 297-304, (1967).

48 Sun, M., Takayama, K., "Vorticity production in shock diffraction," Journal of Fluid Mechanics 478, pp. 237-256, (2003).

49 Takayama, K., Inoue, O., "Shock wave diffraction over a 90 degree sharp corner - posters presented at 18th ISSW," Shock Waves 1, pp. 301-312, (1991)

50 Weigand, A., Gharib, M., "On the decay of a turbulent vortex ring," Physics of Fluids 6 , pp. 3806-3808, (1994).

51 Menon, N., Skews, B. W., "Effect of nozzle inlet geometry on underexpanded supersonic jet characteristics," 26th International Symposium on Shock Waves (ISSW26), Göttingen, Paper 1020, (2007).

52 Menon, N., Skews, B. W., "Rectangular underexpanded gas jets: effect of pressure ratio, aspect ratio and Mach number," 26th International Symposium on Shock Waves (ISSW26), Göttingen, Paper 1021, (2007).

53 Ellzey, J. L., Henneke, M. R., Picone, J. M., Oran, E. S., "The interaction of a shock with a vortex: Shock distortion and the production of acoustic waves," Physics of Fluids 7, pp. 172-184, (1995).

54 James, S., Madnia, C. K., "Direct numerical simulation of a laminar vortex ring," Physics of Fluids 8, pp. 2400-2414, (1996).

55 Maxworthy, T., "The structure and stability of vortex rings," Journal of Fluid Mechanics 51, pp. 15-32, (1972).

56 Golub, V. V., "Development of shock wave and vortex ring structures in unsteady jets," Shock Waves 3, pp. 279-285, (1994).

${ }^{57}$ Abate, G., Shyy, W., "Dynamic structure of confined shocks undergoing sudden expansion," Progress in Aerospace Sciences 38, pp. 23-42, (2002).

58 Chatterjee, A., "Shock wave deformation in shock-vortex interactions," Shock Waves 9, pp. 
95-105, (1999).

59 Sullivan, J. P., Widnall, S. E., Ezekiel, S., "Study of vortex rings using a laser doppler velocimeter," AIAA Journal 11, pp. 1384-1389, (1973).

60 Widnall, S. E., Sullivan, J. P., "On the stability of vortex rings," Proceedings of the Royal Society of London, Series A 332, pp. 335-353, (1973).

61 Wang, X. K., Yu, S. C. M., Chua, L. P., "Effect of delta tabs on axis switching of a coaxial square jet," Journal of Propulsion and Power 20, pp. 533-541, (2004).

62 Broadbent, E. G., Moore, D. W., "The interaction of a vortex ring and a coaxial supersonic jet," Proceedings of the Royal Society of London, Series A 409, pp. 47-57, (1987).

63 Sun, M., Takayama, K., "The formation of a secondary shock wave behind a shock wave diffracting at a convex corner," Shock Waves 7, pp. 287-295, (1997).

64 Addy, A. L., "Effects of axisymmetric sonic nozzle geometry on Mach disk characteristics," AIAA Journal 19, pp. 121-122, (1981).

65 Archer, P. J., Thomas, T. G., Coleman, G. N., "Direct numerical simulation of vortex ring evolution from the laminar to the early turbulent regime," Journal of Fluid Mechanics 598, pp. 201-226, (2008).

66 Bergdorf, M., Koumoutsakos, P., Leonard, A., "Direct numerical simulation of vortex rings at $R_{\Gamma}=7500$," Journal of Fluid Mechanics 581, pp. 495-505, (2007).

67 Viets, H., Sforza, P. M., "Dynamics of bilaterally symmetric vortex rings," Physics of Fluids 15, pp. 230-240, (1972).

68 Lugt, H. J., "Vortex Flows in Nature and Technology," John Wiley \& Sons, (1983). 
TABLE I: Experimental conditions corresponding to the various nozzles

\begin{tabular}{c|c|c|c|c}
\hline & $\begin{array}{c}M_{s e} \\
\text { Experiment }\end{array}$ & $\begin{array}{c}M_{s e} \\
\text { Theory }\end{array}$ & $\Delta M(\%)$ & $R e\left(\times 10^{5}\right)$ \\
\hline Ellipse 0.4 & 1.10 & 1.44 & 31 & 0.52 \\
Ellipse 0.4 & 1.32 & 1.67 & 26 & 1.67 \\
Ellipse 0.4 & 1.41 & 1.83 & 30 & 2.42 \\
\hline Ellipse 0.6 & 1.09 & 1.37 & 26 & 0.7 \\
Ellipse 0.6 & 1.22 & 1.58 & 29 & 1.78 \\
Ellipse 0.6 & 1.37 & 1.72 & 25 & 3.2 \\
\hline Circle & 1.17 & 2.09 & 79 & 1.14 \\
Circle & 1.58 & 2.57 & 63 & 4.57 \\
Circle & 1.62 & 2.86 & 76 & 4.93 \\
\hline Square & 1.05 & 1.28 & 22 & 0.63 \\
Square & 1.21 & 1.44 & 19 & 2.82 \\
Square & 1.43 & 1.56 & 9 & 6.34 \\
\hline
\end{tabular}




\section{List of Figures}

1 Schematic diagram of the schlieren photography setup. . . . . . . . . . . 27

2 Schematic of the PIV setup. . . . . . . . . . . . . . . . . 27

3 Features of a shock diffraction pattern around a corner. . . . . . . . . . . . 28

4 Elliptic nozzle (0.4), oblique view, 4 bar, $t=$ (a) $0.1 \mathrm{~ms}$, (b) $0.3 \mathrm{~ms}$. . . . 28

5 Elliptic nozzle (0.4), side view, major axis horizontal, 4 bar, $t=$ (a) $0.4 \mathrm{~ms}$, (b) $0.63 \mathrm{~ms} \ldots \ldots \ldots \ldots$

6 PIV results for elliptic nozzle (0.4), side view, major axis horizontal, 4 bar, $t=$ (a) $0.14 \mathrm{~ms}$, (b) $0.27 \mathrm{~ms}$, (c) $0.44 \mathrm{~ms}$. . . . . . . . . . . . . . . . . . 29

7 Elliptic nozzle (0.4), side view, 8 bar, $t=(\mathrm{a}) \&(\mathrm{~b}) 0.1 \mathrm{~ms}$, (c) \& (d) $0.18 \mathrm{~ms}$, (e) \& (f) $0.25 \mathrm{~ms} \ldots \ldots \ldots$. . . . . . . . . . . . . . . . 30

8 PIV results for elliptic nozzle (0.4), side view, major axis horizontal, 8 bar, $t=$ (a) $0.15 \mathrm{~ms}$, (b) $0.27 \mathrm{~ms}$, (c) $0.59 \mathrm{~ms}$, (d) $0.61 \mathrm{~ms}$. . . . . . . . . . . 31

9 Elliptic nozzle (0.4), side view, major axis horizontal, 12 bar, $t=0.32 \mathrm{~ms} . \quad$. 32

10 Elliptic nozzle (0.4) variation of: (a) vortex loop diameter, (b) vortex loop

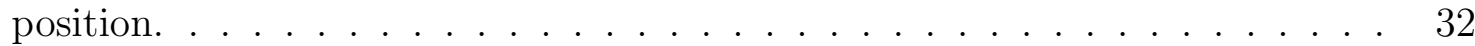

11 Elliptic nozzle $(0.6)$, oblique view, 4 bar, $t=0.5 \mathrm{~ms}$. . . . . . . . . . . 32

12 PIV results for elliptic nozzle (0.6), side view, major axis horizontal, 4 bar, $t=$ (a) $0.51 \mathrm{~ms}$, (b) $0.72 \mathrm{~ms}$.

13 PIV results for elliptic nozzle (0.6), side view, major axis horizontal, 8 bar, $t=$ (a) $0.36 \mathrm{~ms}$, (b) $0.42 \mathrm{~ms}$.

14 Elliptic nozzle (0.6), side view, $12 \mathrm{bar}, t=$ (a) \& (b) $0.11 \mathrm{~ms}$, (c) \& (d)

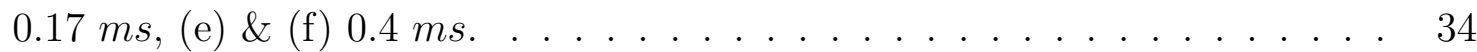

15 PIV results for elliptic nozzle (0.6), side view, major axis horizontal, 12 bar, $t=$ (a) $0.06 \mathrm{~ms}$, (b) $0.14 \mathrm{~ms}$, (c) $0.18 \mathrm{~ms}$, (d) $0.48 \mathrm{~ms} . \ldots \ldots$. . . . . .

16 Elliptic nozzle (0.6) variation of: (a) vortex loop diameter, (b) vortex loop

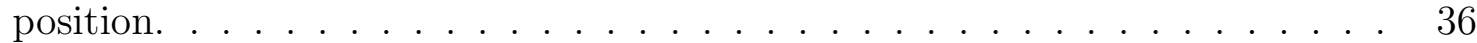

17 Circular nozzle, side view, 8 bar, $t=$ (a) $0.17 \mathrm{~ms}$, (b) $0.21 \mathrm{~ms}$, (c) $0.27 \mathrm{~ms}$,

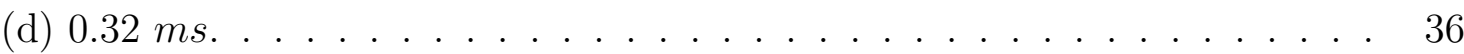

18 PIV results for circular nozzle, side view, 8 bar, $t=$ (a) $0.12 \mathrm{~ms}$, (b) $0.22 \mathrm{~ms}$,

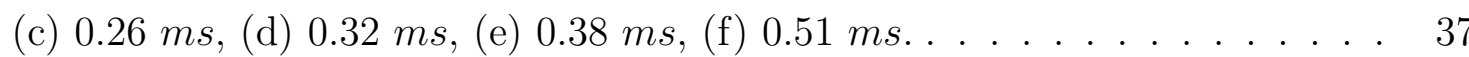


19 Circular nozzle, oblique view, 12 bar, $t=$ (a) $0.13 \mathrm{~ms}$, (b) $0.19 \mathrm{~ms}$, (c) $0.22 \mathrm{~ms} .38$

20 PIV results for circular nozzle, side view, $12 \mathrm{bar}, t=$ (a) $0.09 \mathrm{~ms}$, (b) $0.13 \mathrm{~ms}$,

(c) $0.18 \mathrm{~ms}$, (d) $0.24 \mathrm{~ms}$, (e) $0.32 \mathrm{~ms}$, (f) $0.44 \mathrm{~ms} \ldots \ldots$. . . . . . . . . . . . . 39

21 Circular nozzle variation of: (a) vortex loop diameter, (b) vortex loop position. 39

22 Square nozzle, 4 bar, $t=$ (a) \& (b) $0.25 \mathrm{~ms}$, (c) \& (d) $0.45 \mathrm{~ms}$. ((a) and (c) are side views, (b) and (d) are oblique views) . . . . . . . . . . . . 40

23 Schematic of mean streamwise vorticity distributions in a square jet, (a) close to the jet exit, (b) further downstream. ${ }^{61} \ldots \ldots$. . . . . . . . . . 41

24 PIV results for square nozzle, side view, 4 bar, $t=$ (a) $0.16 \mathrm{~ms}$, (b) $0.56 \mathrm{~ms}$,

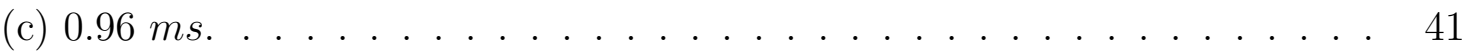

25 PIV results for square nozzle, side view, $12 \mathrm{bar}, t=(\mathrm{a}) 0.22 \mathrm{~ms}$, (b) $0.3 \mathrm{~ms}$, (c) $0.47 \mathrm{~ms}$, (d) $0.53 \mathrm{~ms} \ldots \ldots \ldots$. . . . . . . . . . . . . . . . . . 42

26 Square nozzle variation of: (a) vortex loop diameter, (b) vortex loop position. 43 


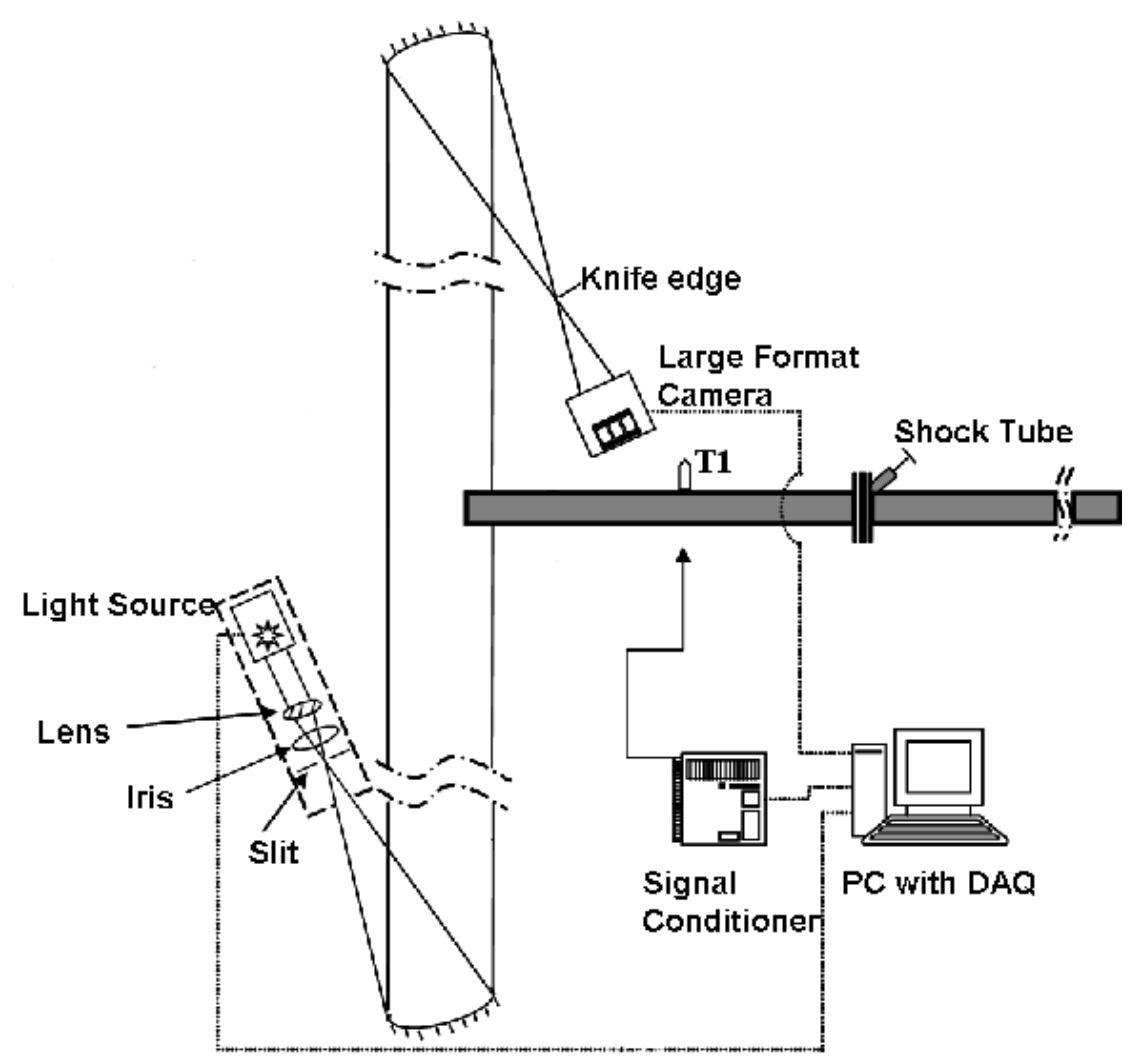

FIG. 1: Schematic diagram of the schlieren photography setup.

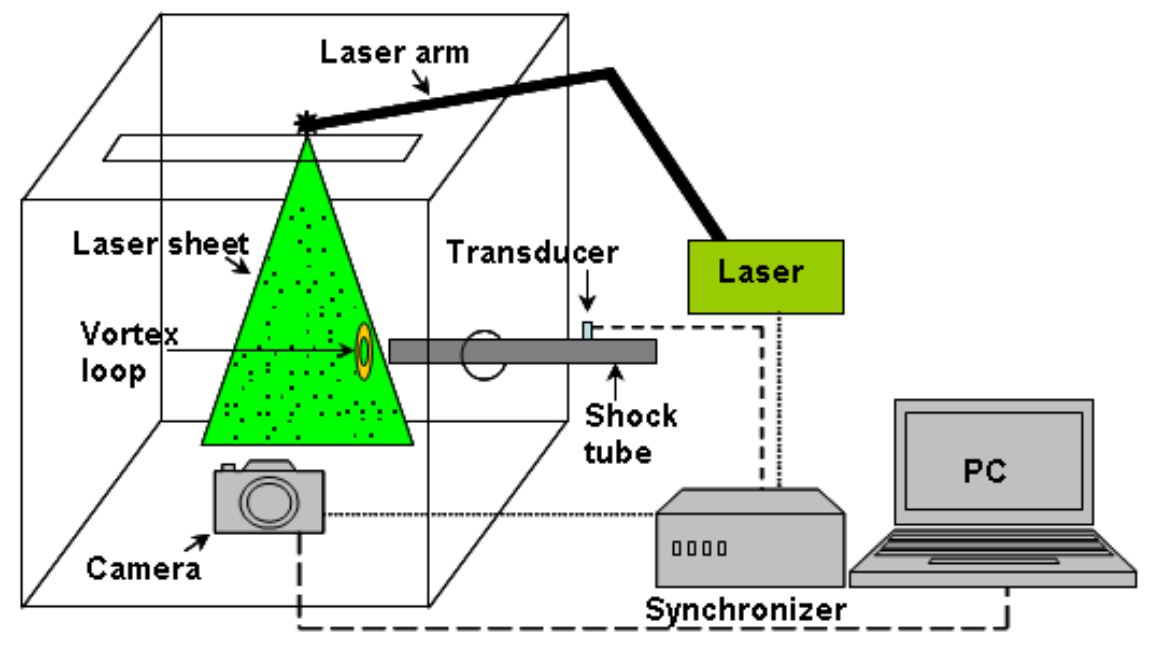

FIG. 2: Schematic of the PIV setup. 


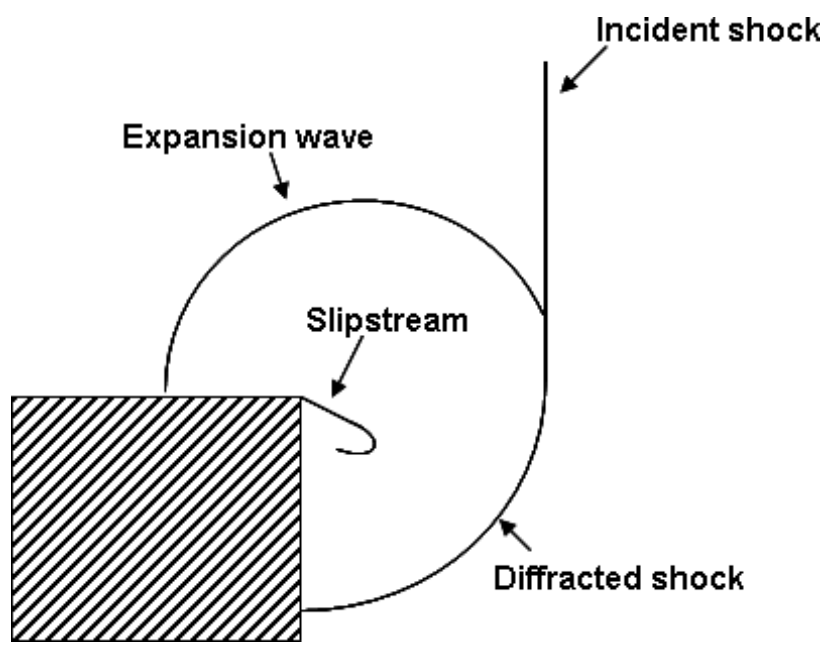

FIG. 3: Features of a shock diffraction pattern around a corner.

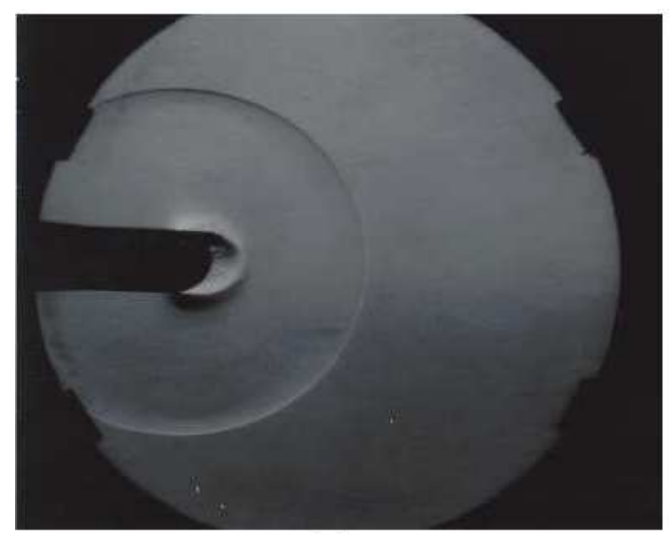

(a)

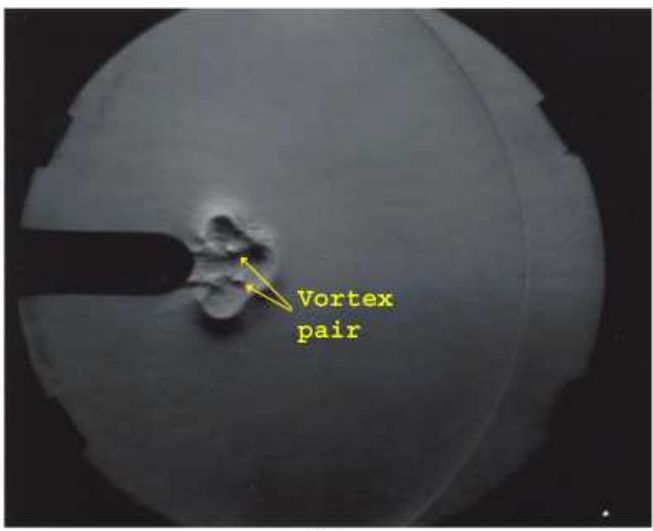

(b)

FIG. 4: Elliptic nozzle (0.4), oblique view, 4 bar, $t=$ (a) $0.1 \mathrm{~ms}$, (b) $0.3 \mathrm{~ms}$.

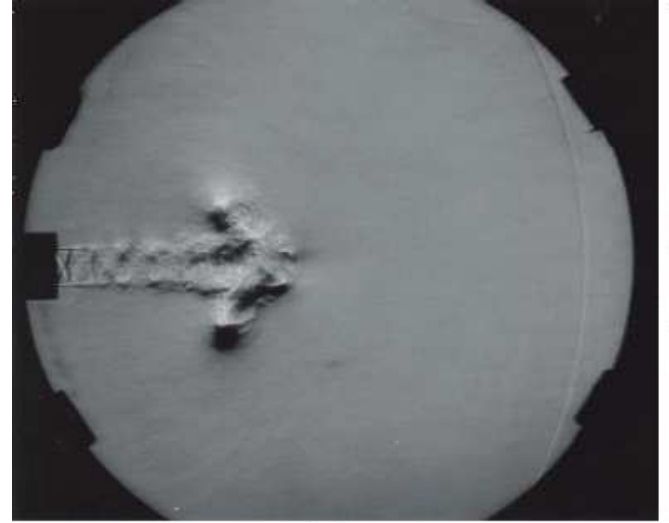

(a)

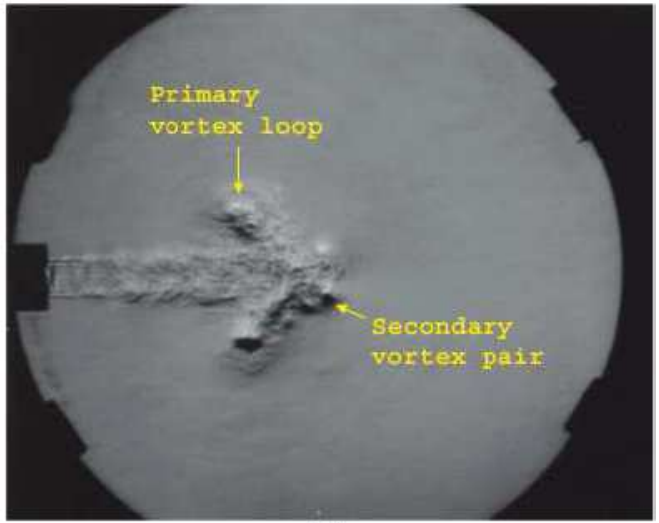

(b)

FIG. 5: Elliptic nozzle (0.4), side view, major axis horizontal, 4 bar, $t=$ (a) $0.4 \mathrm{~ms}$, (b) $0.63 \mathrm{~ms}$. 


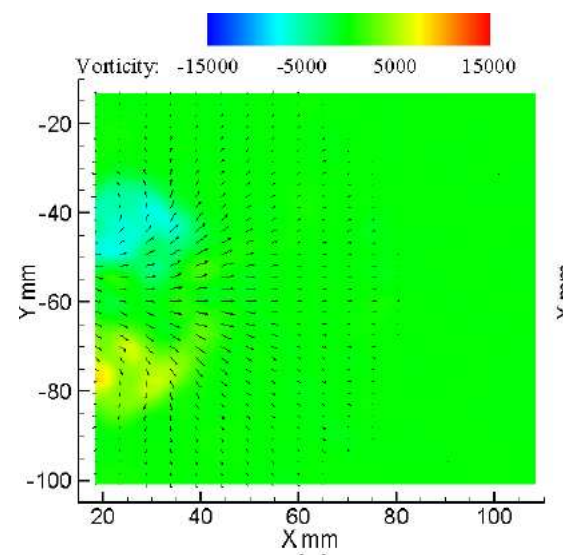

(a)

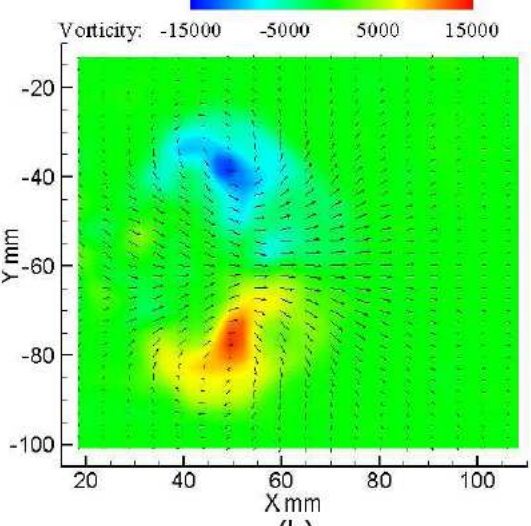

(b)

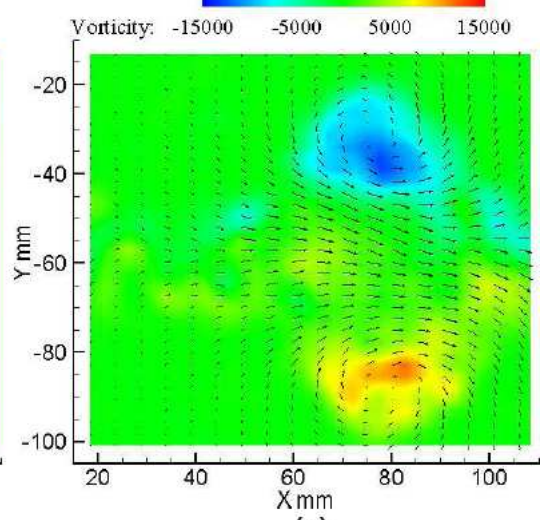

(c)

FIG. 6: PIV results for elliptic nozzle (0.4), side view, major axis horizontal, 4 bar,$t=($ a) $0.14 \mathrm{~ms}$, (b) $0.27 \mathrm{~ms}$, (c) $0.44 \mathrm{~ms}$. 


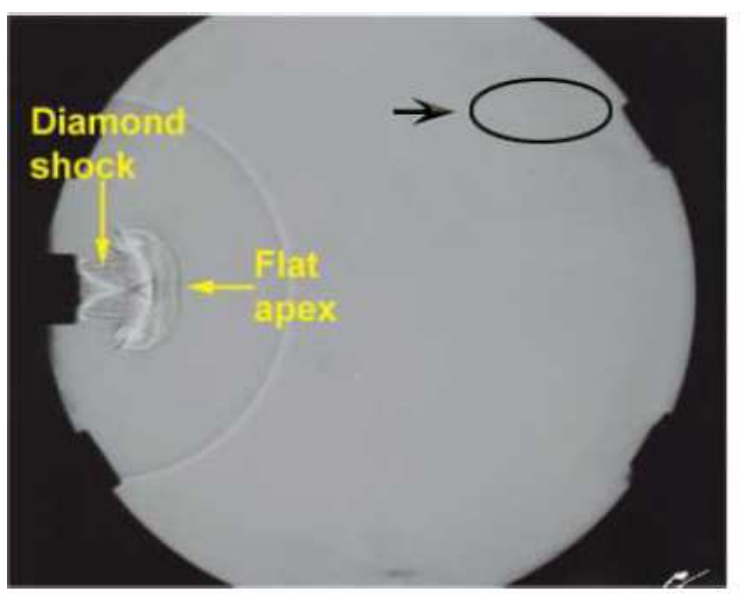

(a)

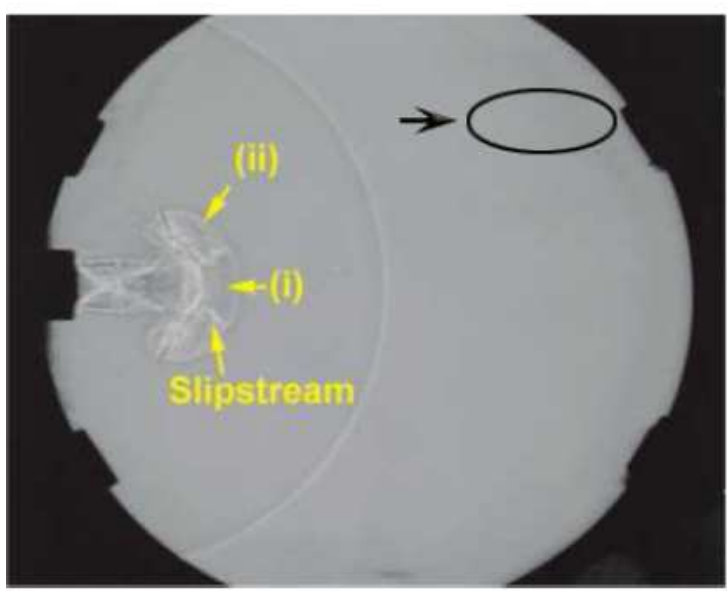

(c)

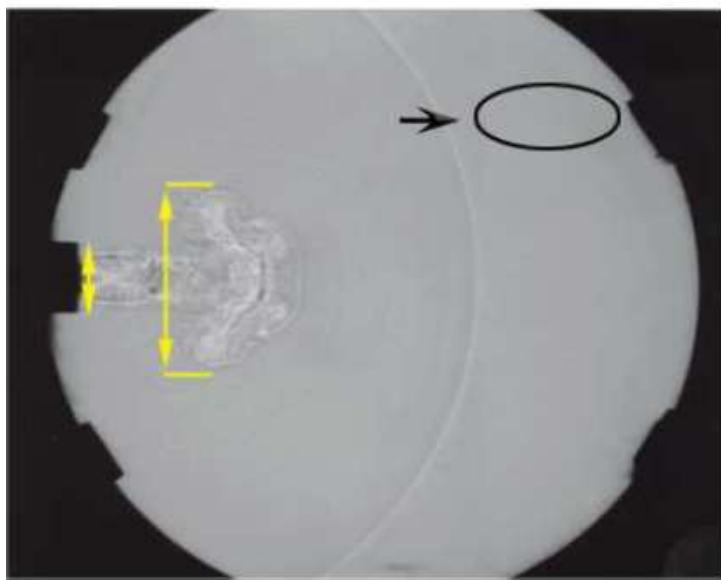

(e)

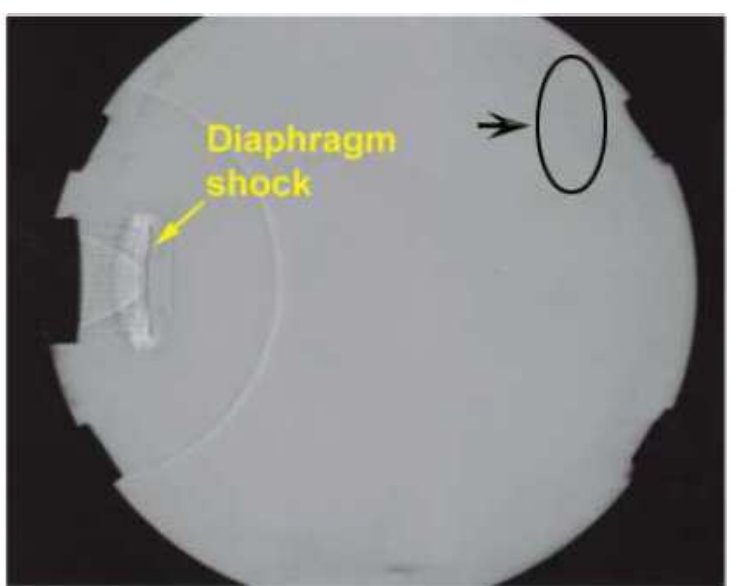

(b)

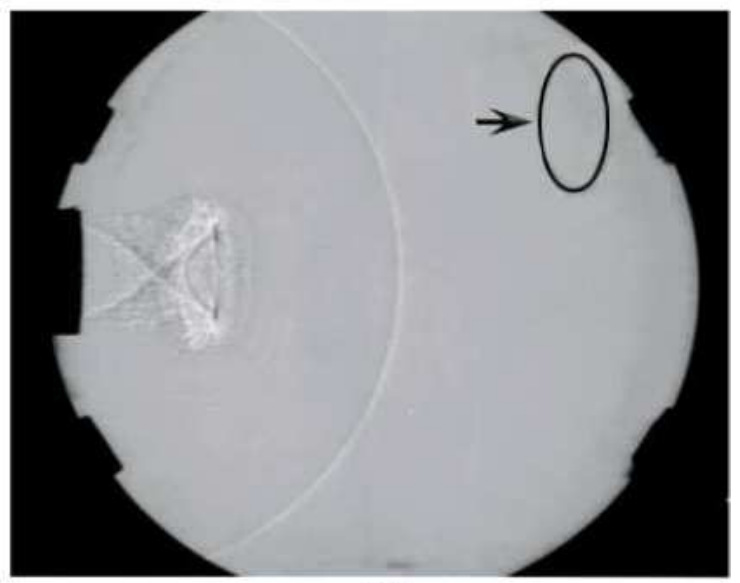

(d)

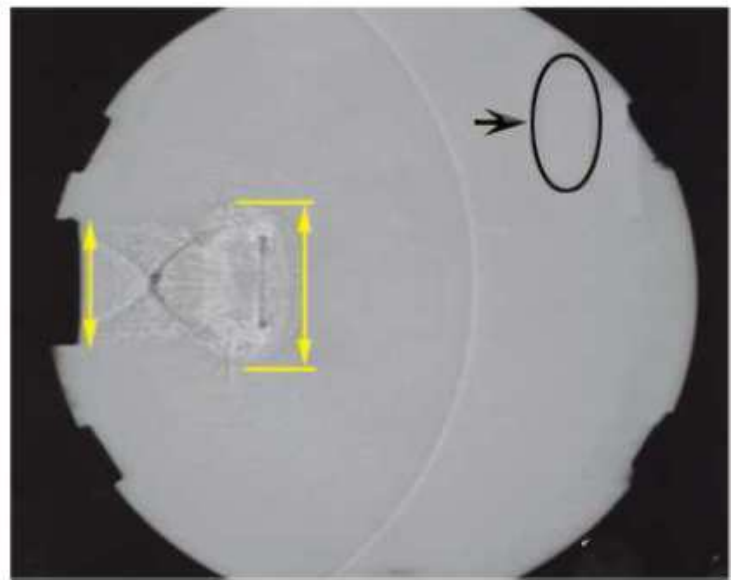

(f)

FIG. 7: Elliptic nozzle (0.4), side view, 8 bar, $t=$ (a) \& (b) $0.1 \mathrm{~ms}$, (c) \& (d) $0.18 \mathrm{~ms}$, (e) \& (f) $0.25 \mathrm{~ms}$. 


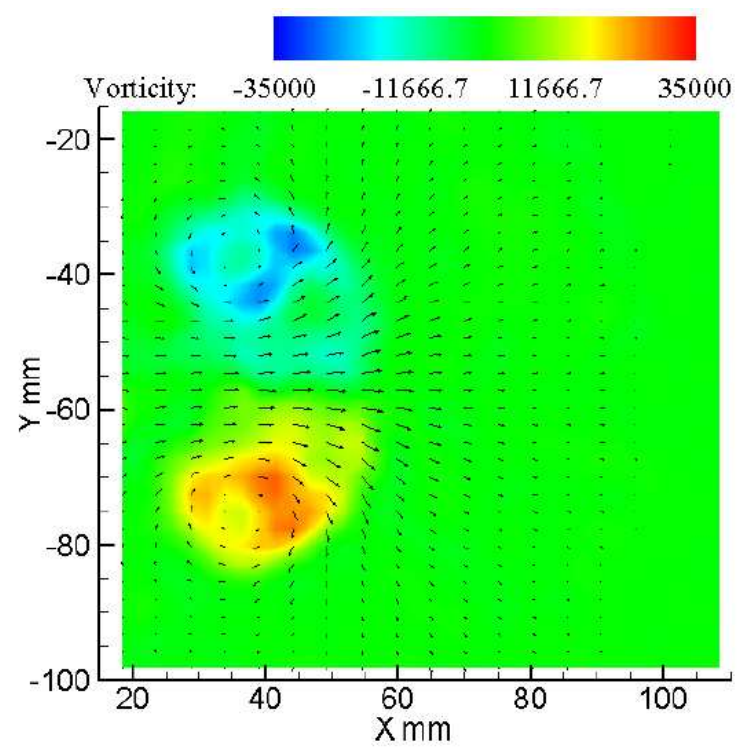

(a)

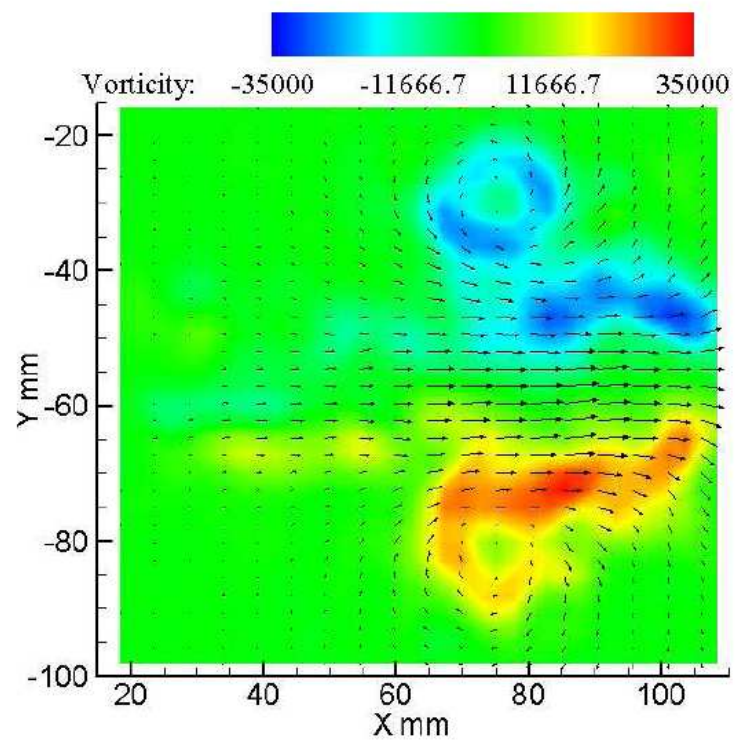

(c)

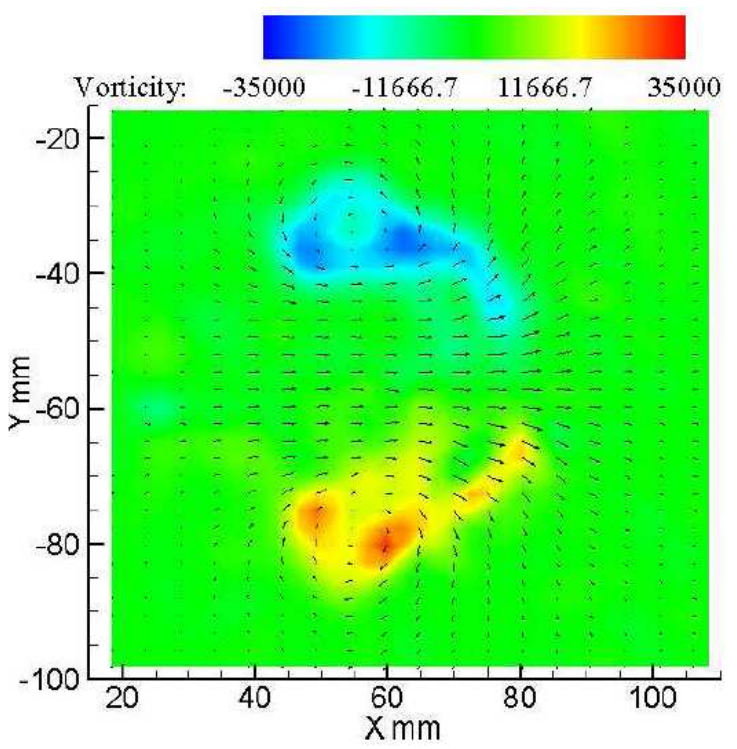

(b)

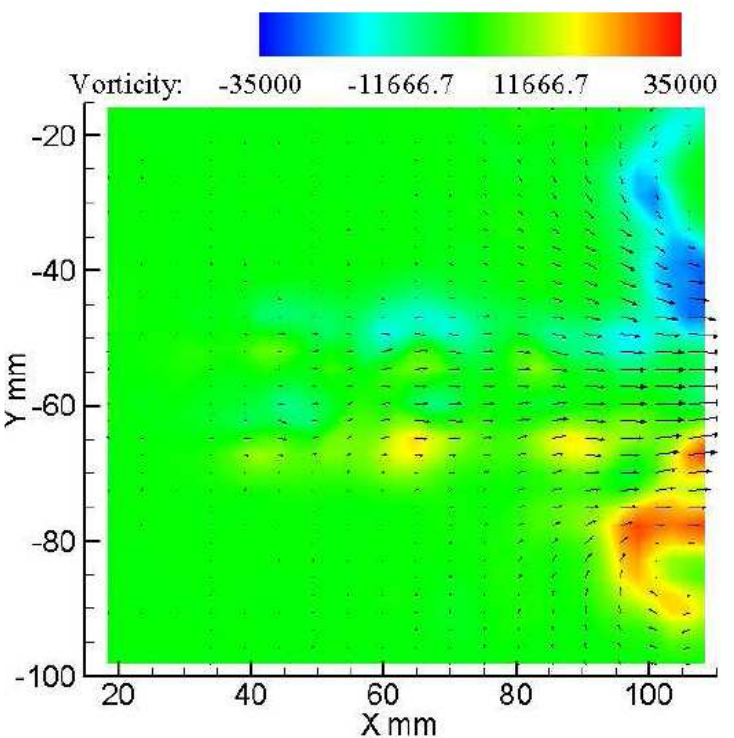

(d)

FIG. 8: PIV results for elliptic nozzle (0.4), side view, major axis horizontal, 8 bar,$t=($ a) $0.15 \mathrm{~ms}$, (b) $0.27 \mathrm{~ms}$, (c) $0.59 \mathrm{~ms}$, (d) $0.61 \mathrm{~ms}$. 


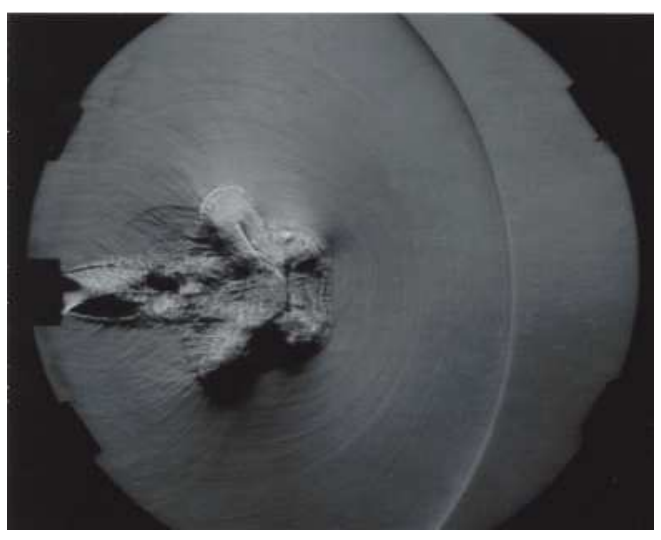

FIG. 9: Elliptic nozzle (0.4), side view, major axis horizontal, 12 bar, $t=0.32 \mathrm{~ms}$.

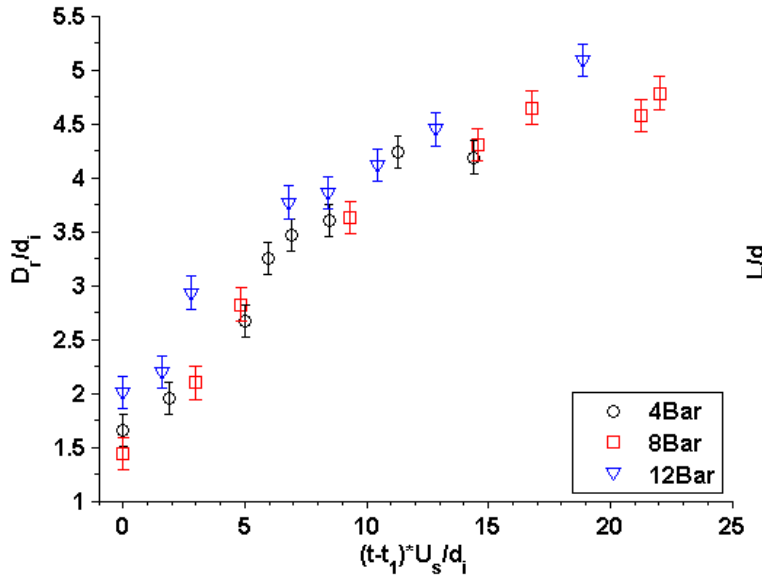

(a)

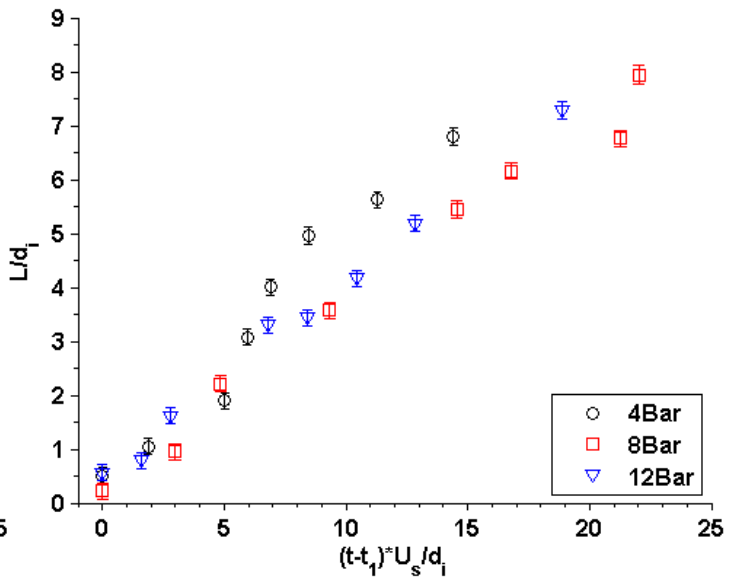

(b)

FIG. 10: Elliptic nozzle (0.4) variation of: (a) vortex loop diameter, (b) vortex loop position.

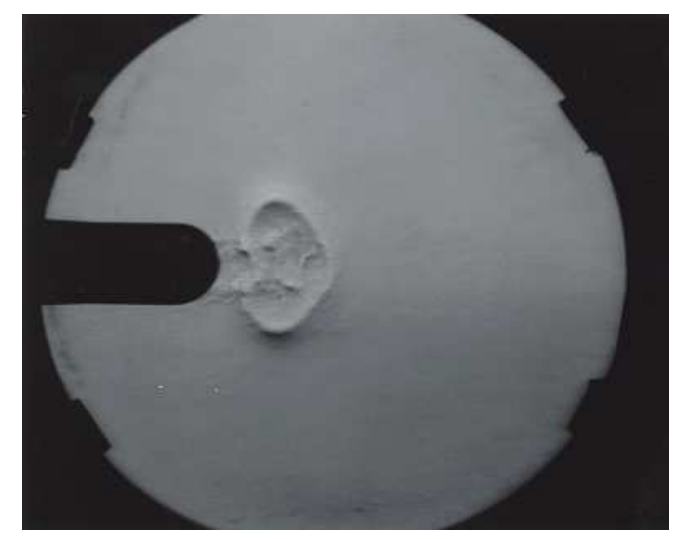

FIG. 11: Elliptic nozzle (0.6), oblique view, 4 bar, $t=0.5 \mathrm{~ms}$. 


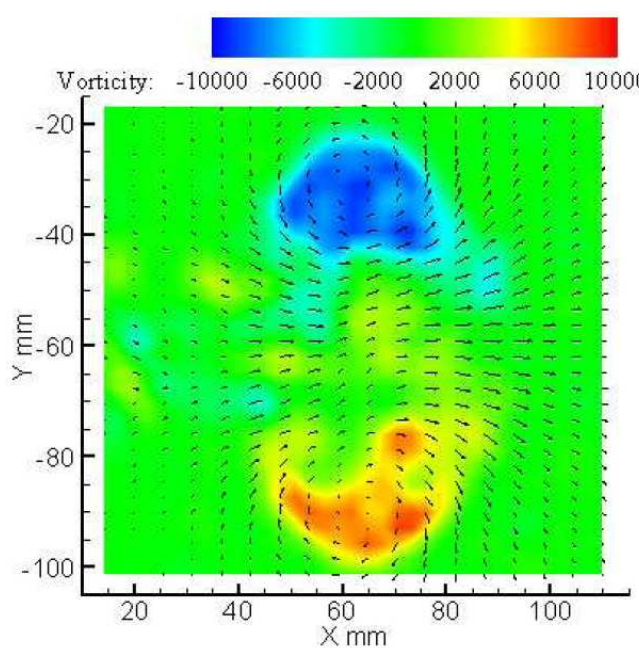

(a)

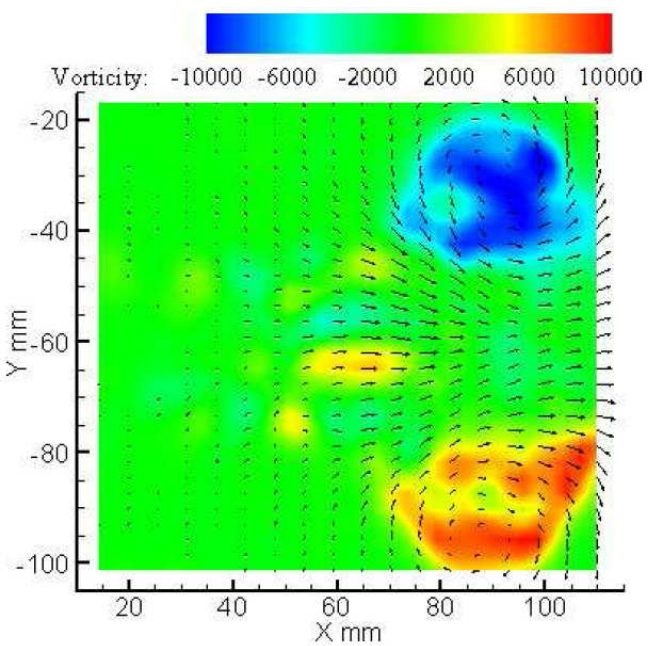

(b)

FIG. 12: PIV results for elliptic nozzle (0.6), side view, major axis horizontal, 4 bar, $t=(\mathrm{a})$ $0.51 \mathrm{~ms}$, (b) $0.72 \mathrm{~ms}$.

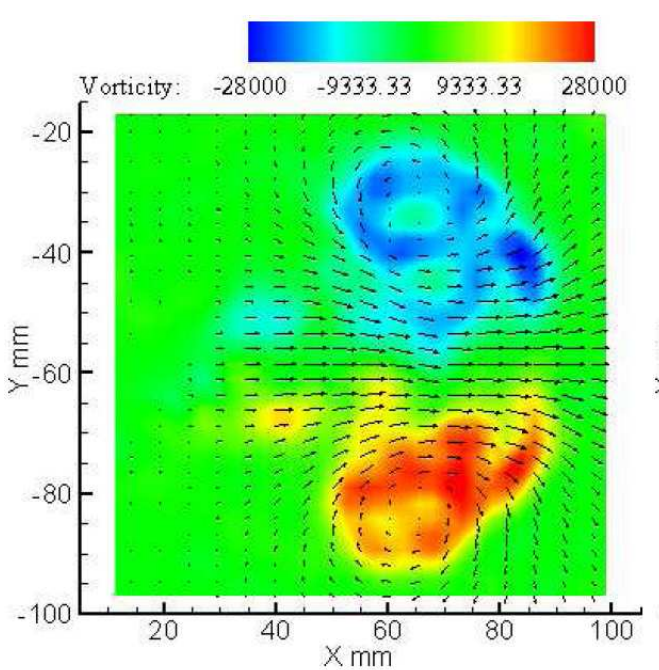

(a)

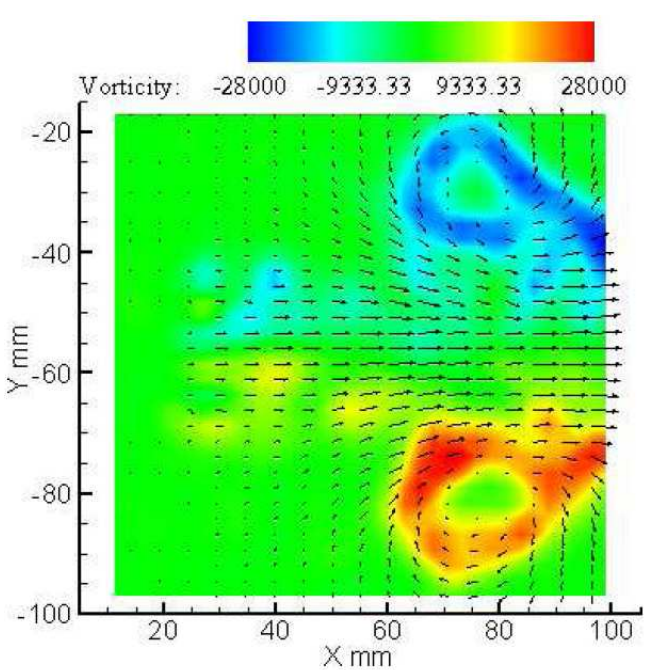

(b)

FIG. 13: PIV results for elliptic nozzle (0.6), side view, major axis horizontal, 8 bar, $t=(\mathrm{a})$ $0.36 \mathrm{~ms}$, (b) $0.42 \mathrm{~ms}$. 


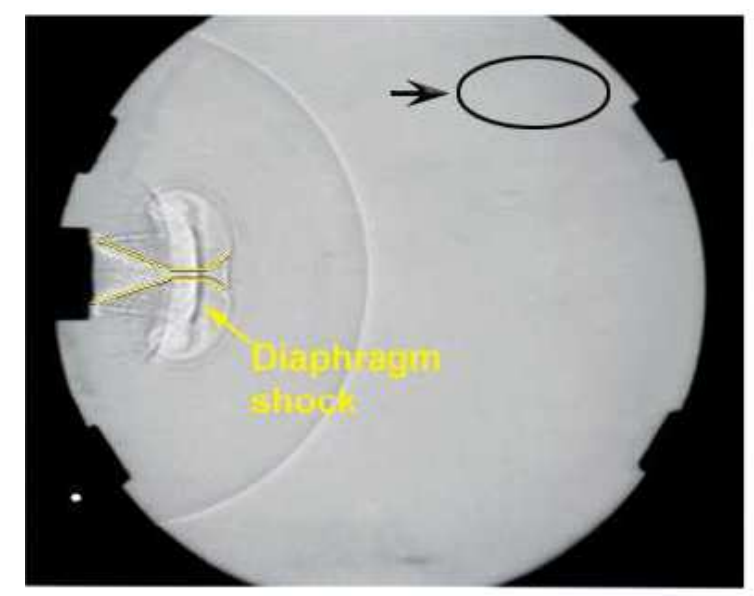

(a)

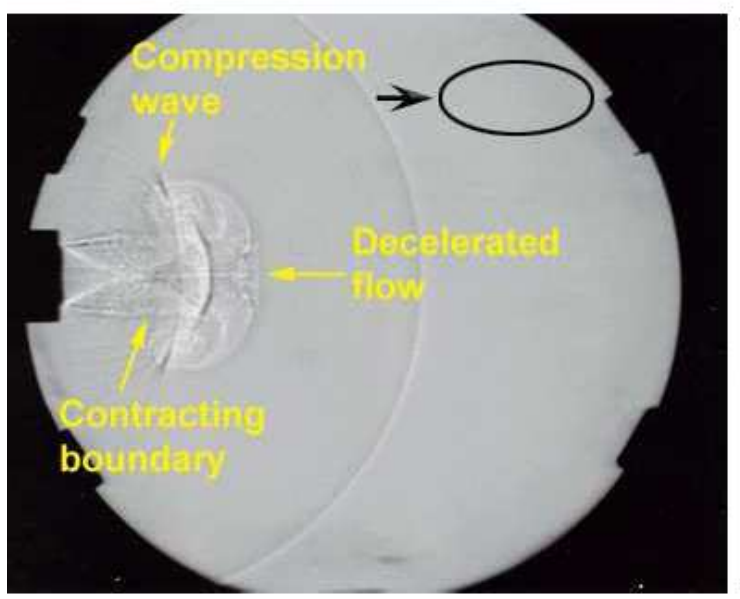

(c)

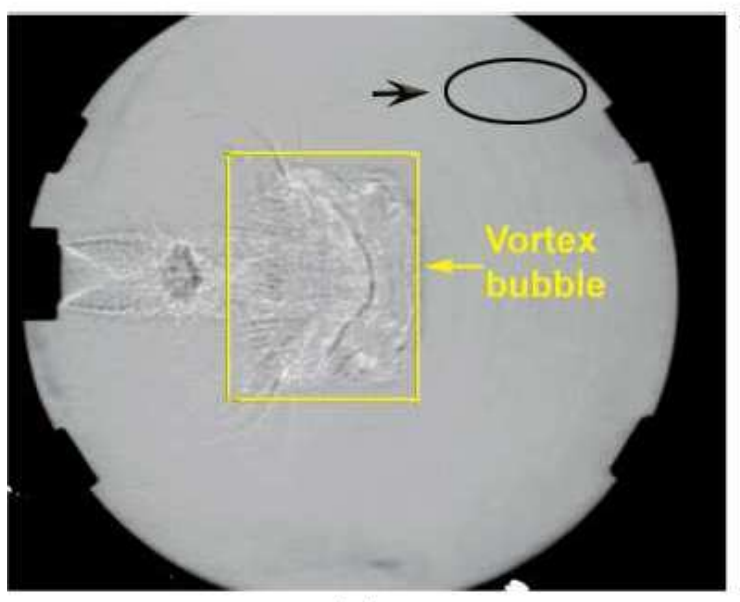

(e)

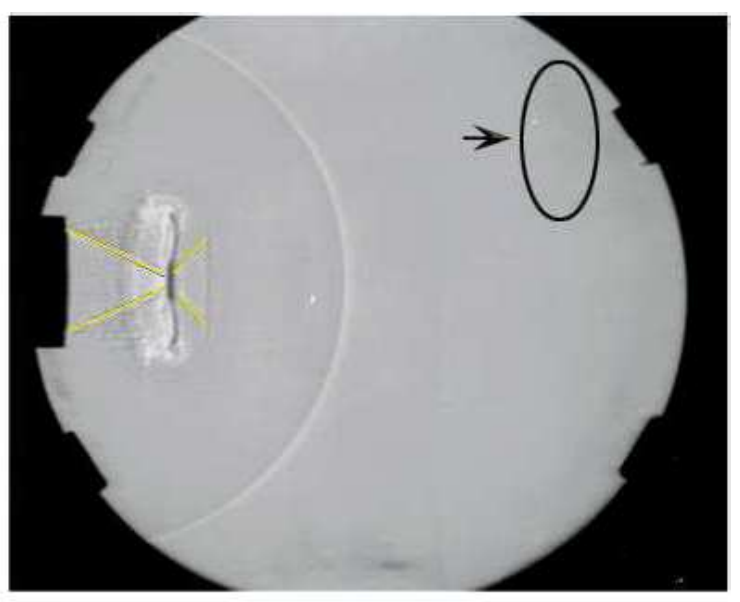

(b)

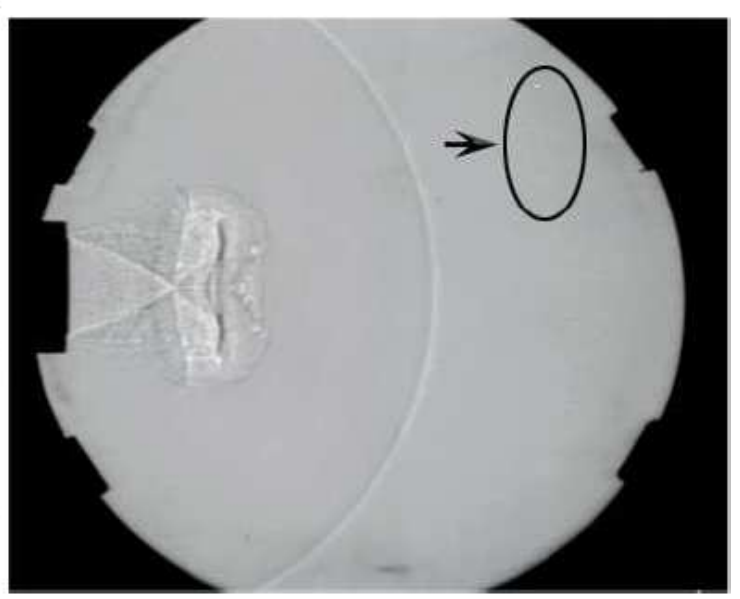

(d)

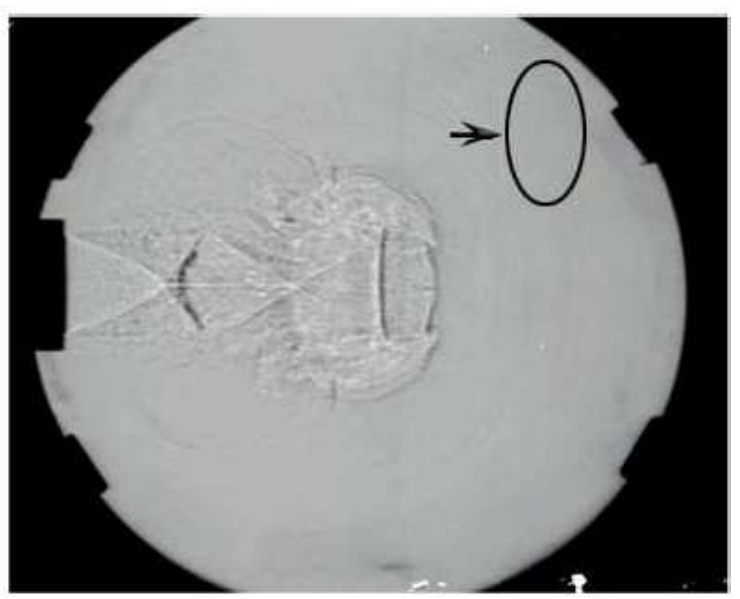

(f)

FIG. 14: Elliptic nozzle (0.6), side view, 12 bar, $t=$ (a) \& (b) $0.11 \mathrm{~ms}$, (c) \& (d) $0.17 \mathrm{~ms}$, (e) \& (f) $0.4 \mathrm{~ms}$. 


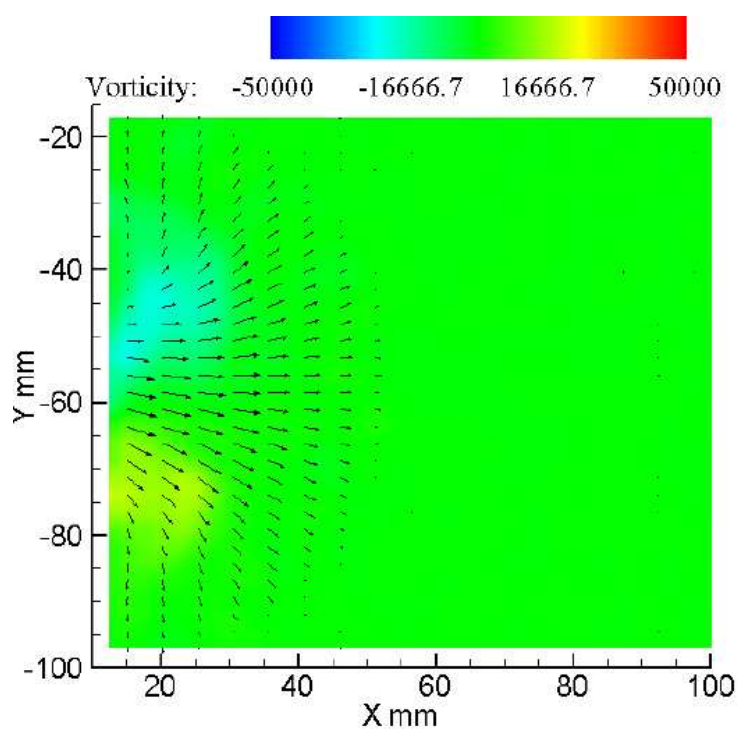

(a)

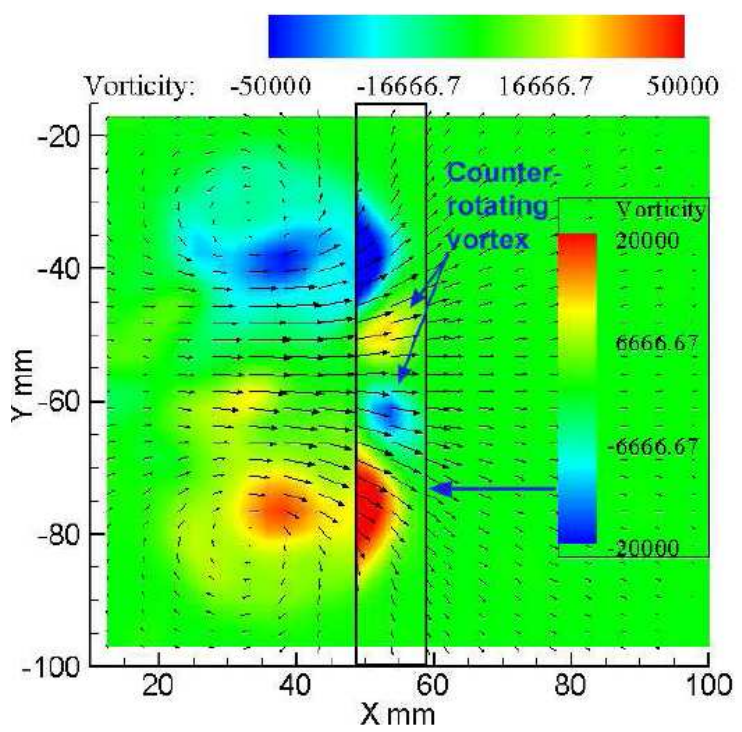

(c)

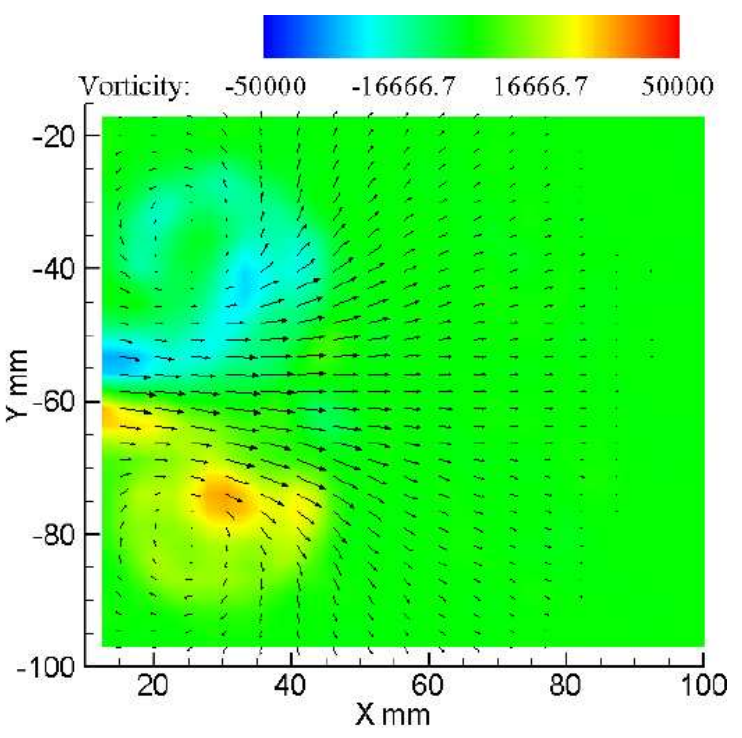

(b)

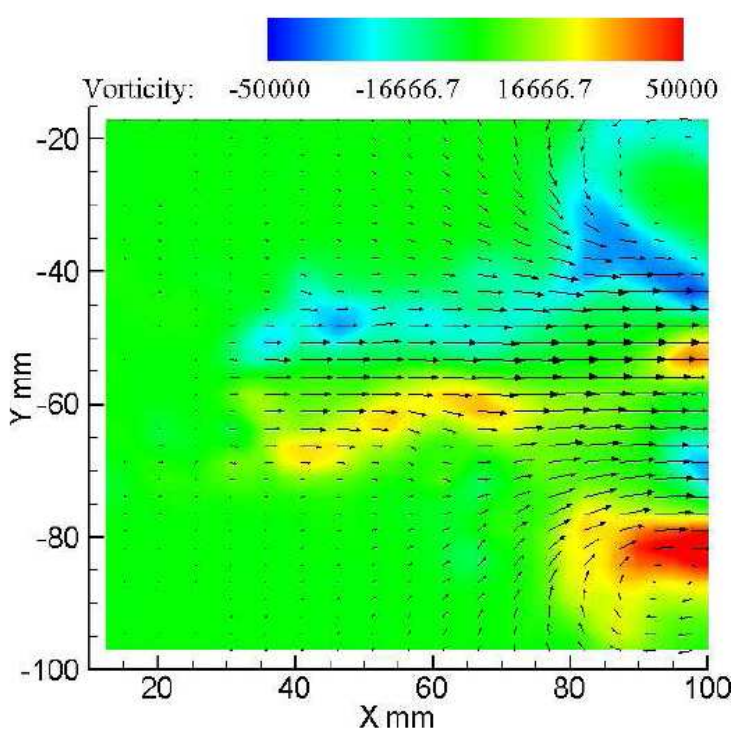

(d)

FIG. 15: PIV results for elliptic nozzle (0.6), side view, major axis horizontal, 12 bar, $t=(\mathrm{a})$ $0.06 \mathrm{~ms}$, (b) $0.14 \mathrm{~ms}$, (c) $0.18 \mathrm{~ms}$, (d) $0.48 \mathrm{~ms}$. 


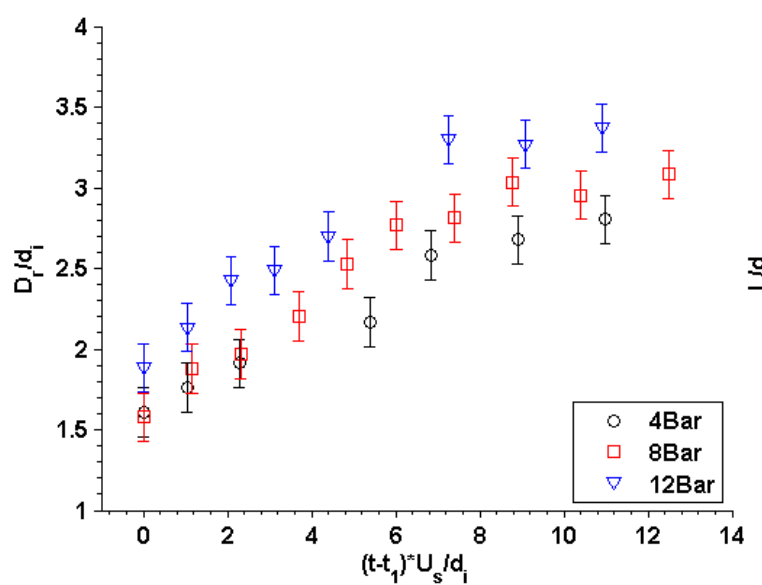

(a)

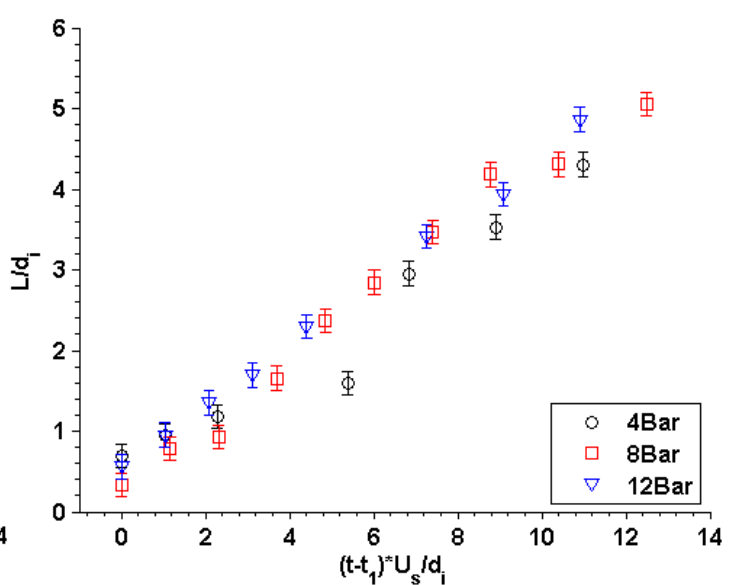

(b)

FIG. 16: Elliptic nozzle (0.6) variation of: (a) vortex loop diameter, (b) vortex loop position.

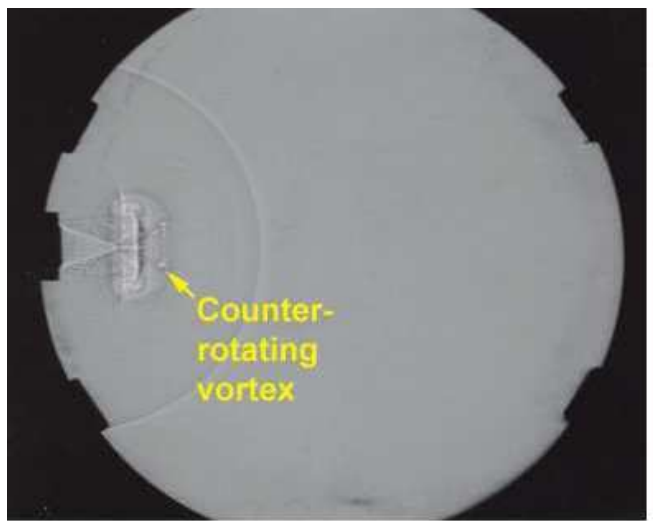

(a)

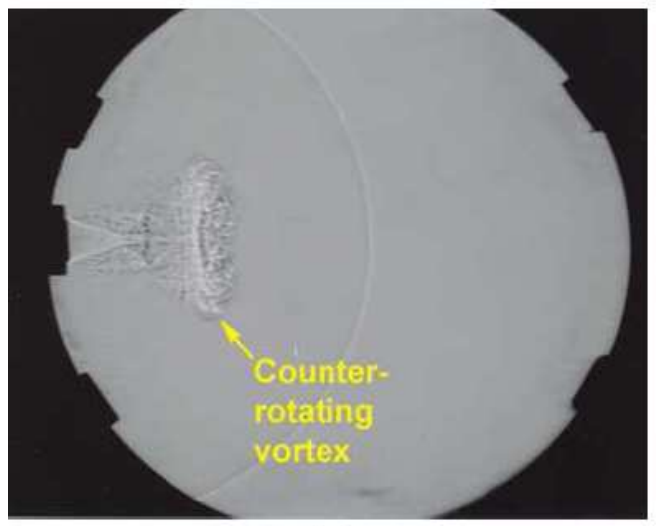

(c)

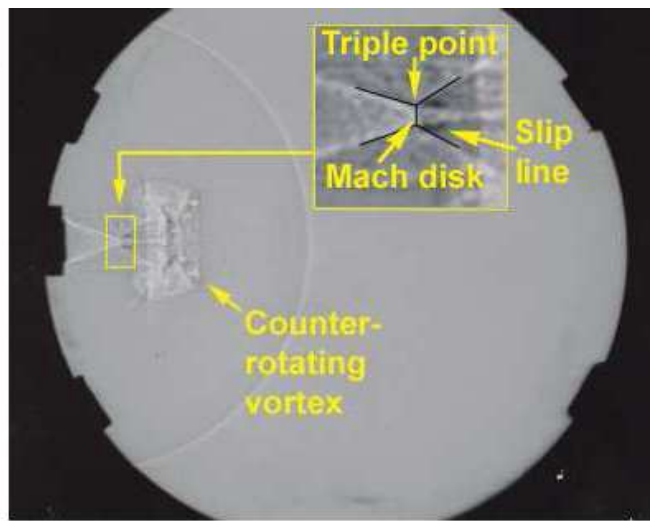

(b)

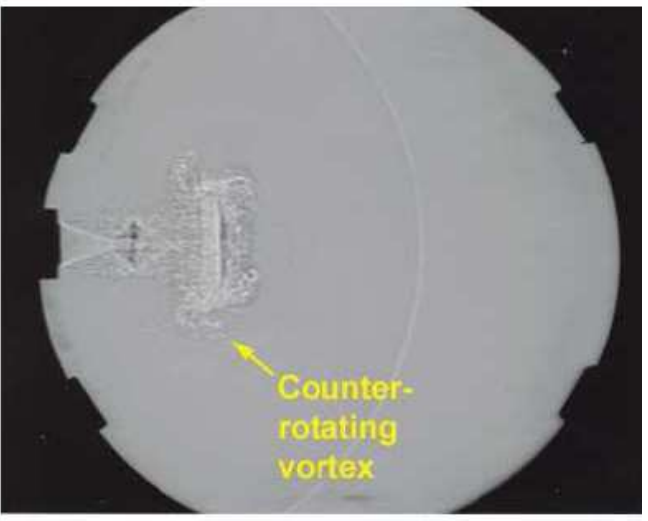

(d)

FIG. 17: Circular nozzle, side view, 8 bar, $t=$ (a) $0.17 \mathrm{~ms}$, (b) $0.21 \mathrm{~ms}$, (c) $0.27 \mathrm{~ms}$, (d) $0.32 \mathrm{~ms}$. 


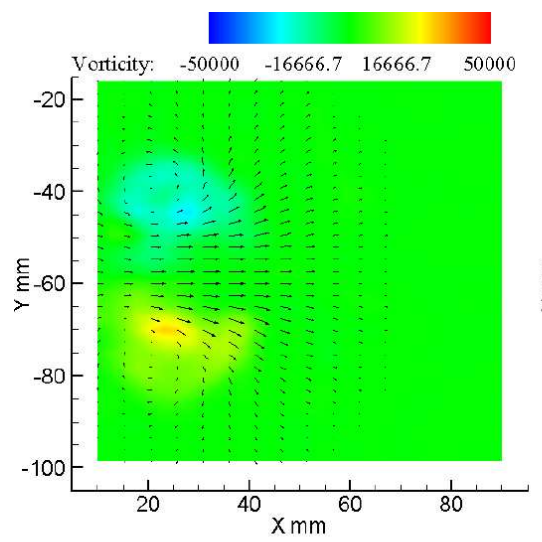

(a)

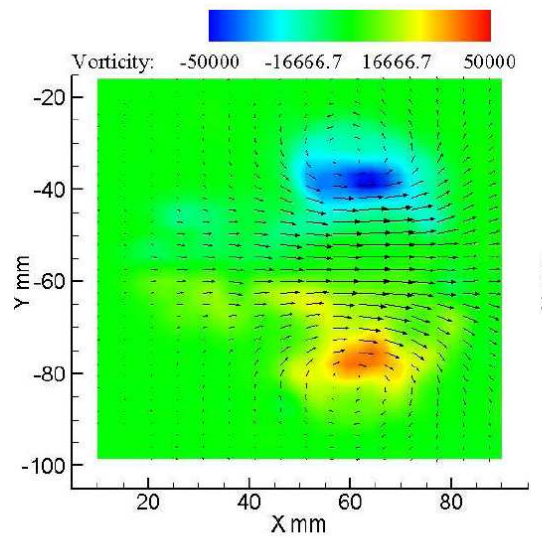

(d)

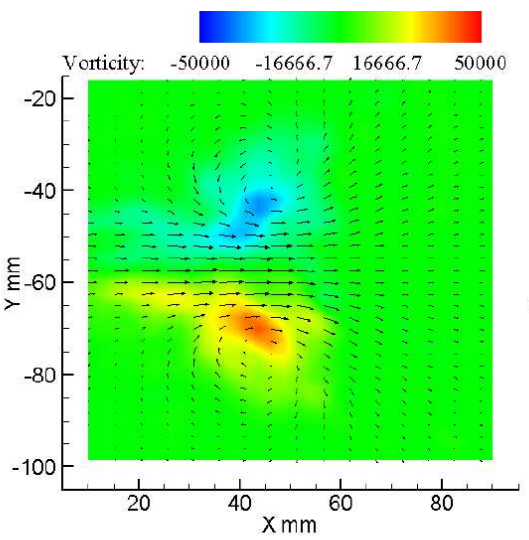

(b)

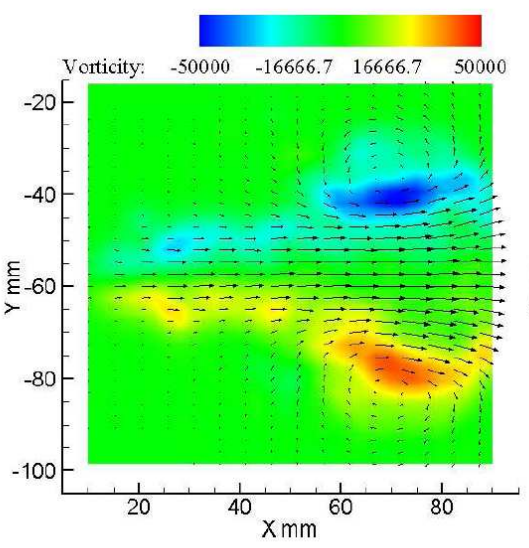

(e)

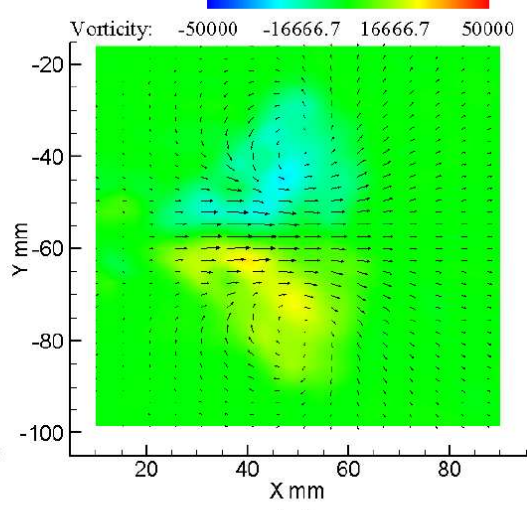

(c)

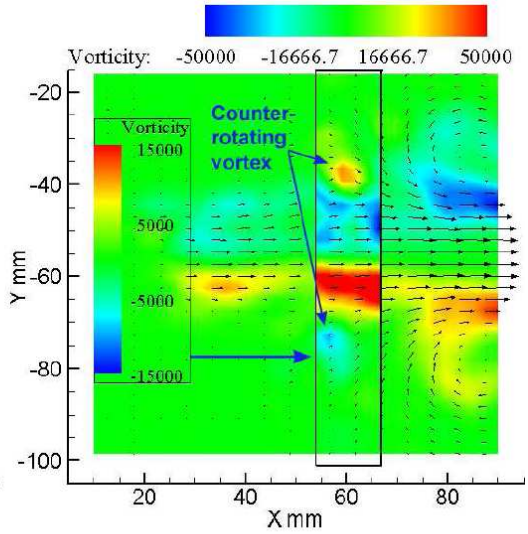

(f)

FIG. 18: PIV results for circular nozzle, side view, 8 bar, $t=$ (a) $0.12 \mathrm{~ms}$, (b) $0.22 \mathrm{~ms}$, (c) $0.26 \mathrm{~ms}$, (d) $0.32 \mathrm{~ms}$, (e) $0.38 \mathrm{~ms}$, (f) $0.51 \mathrm{~ms}$. 


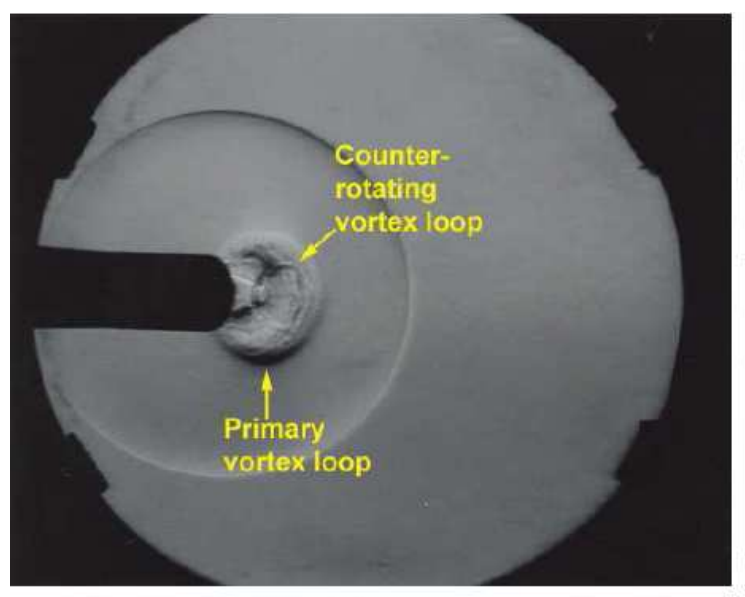

(a)

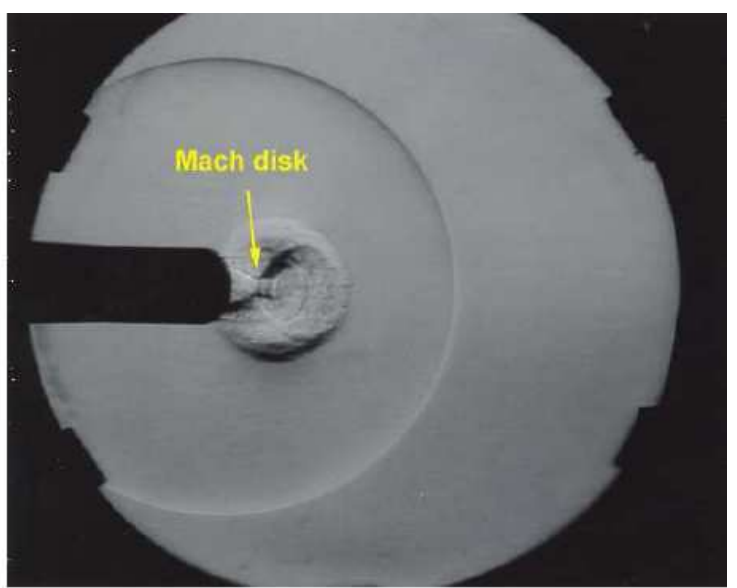

(b)

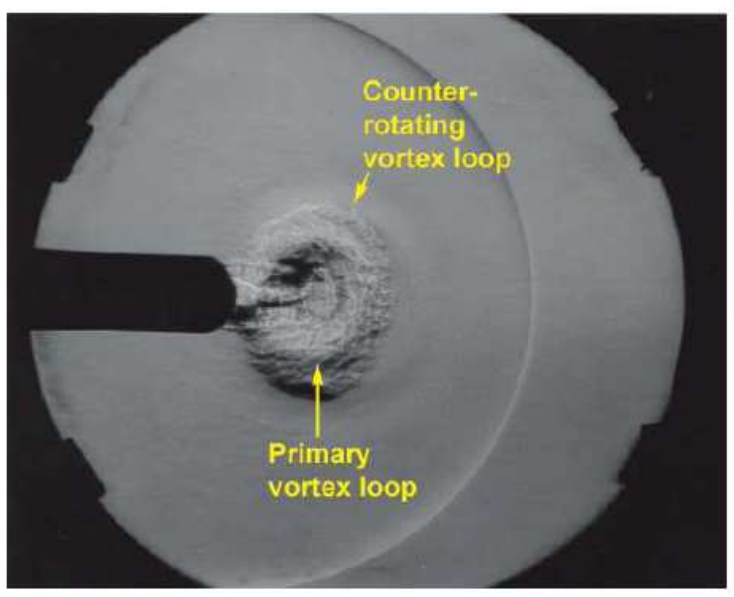

(c)

FIG. 19: Circular nozzle, oblique view, 12 bar, $t=$ (a) $0.13 \mathrm{~ms}$, (b) $0.19 \mathrm{~ms}$, (c) $0.22 \mathrm{~ms}$. 


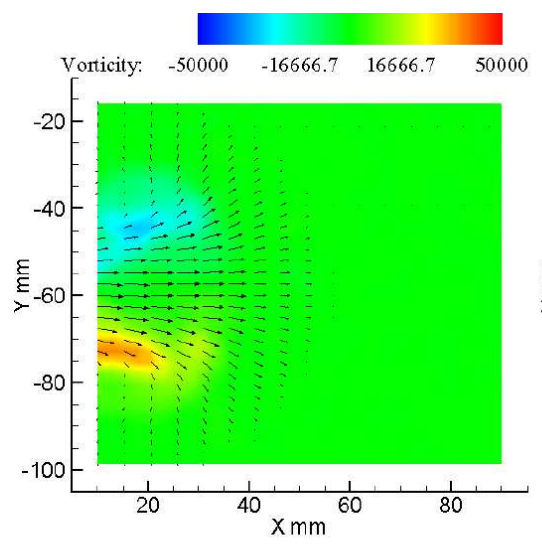

(a)

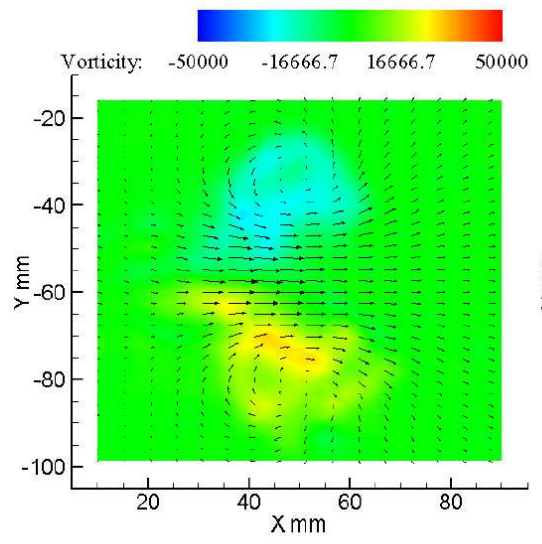

(d)

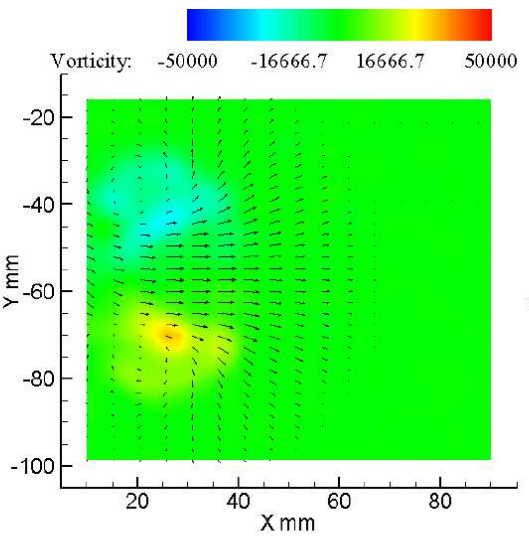

(b)

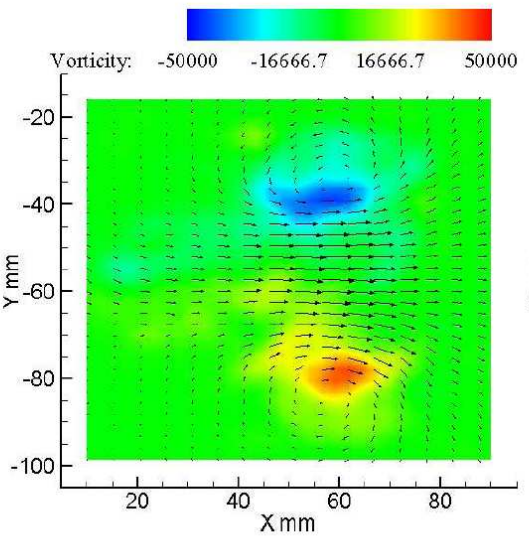

(e)

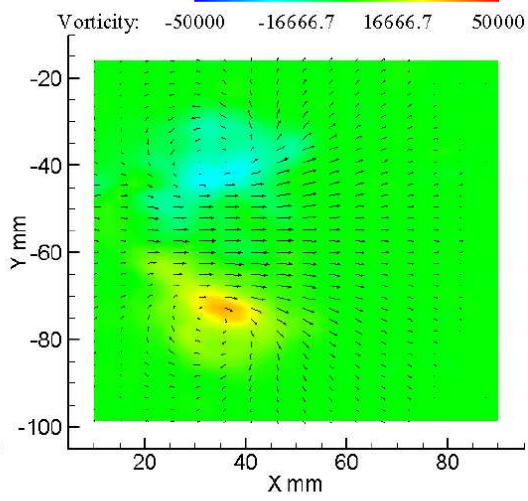

(c)

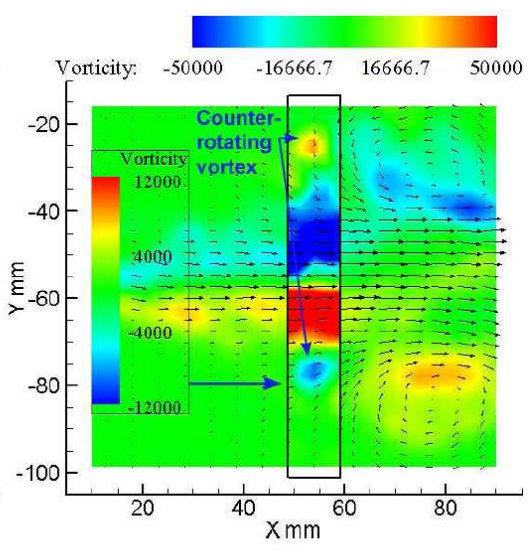

(f)

FIG. 20: PIV results for circular nozzle, side view, $12 \mathrm{bar}, t=$ (a) $0.09 \mathrm{~ms}$, (b) $0.13 \mathrm{~ms}$, (c) $0.18 \mathrm{~ms}$, (d) $0.24 \mathrm{~ms}$, (e) $0.32 \mathrm{~ms}$, (f) $0.44 \mathrm{~ms}$.

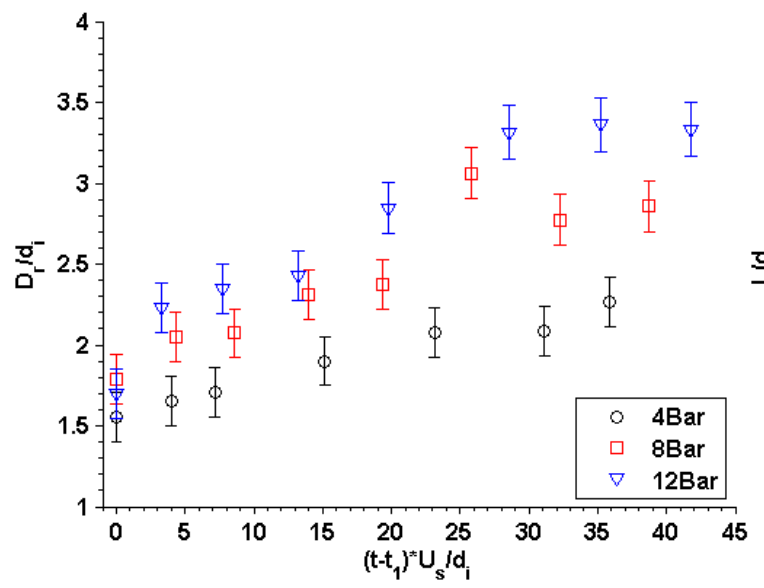

(a)

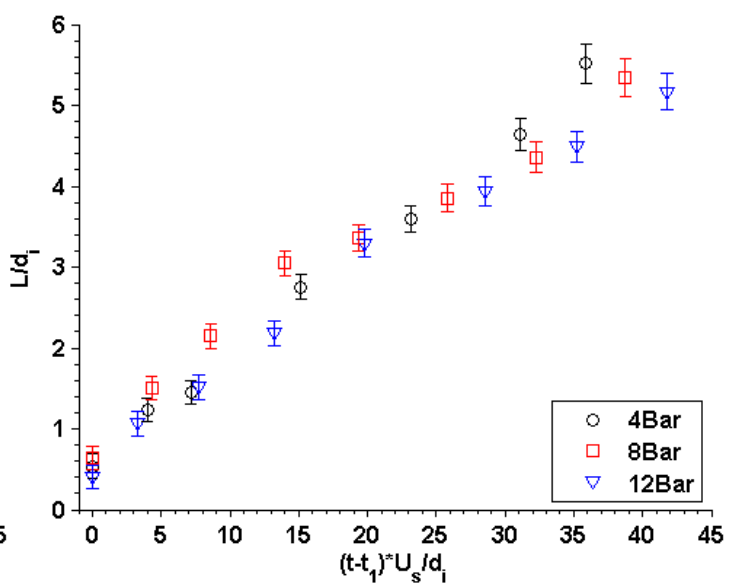

(b)

FIG. 21: Circular nozzle variation of: (a) vortex loop diameter, (b) vortex loop position. 


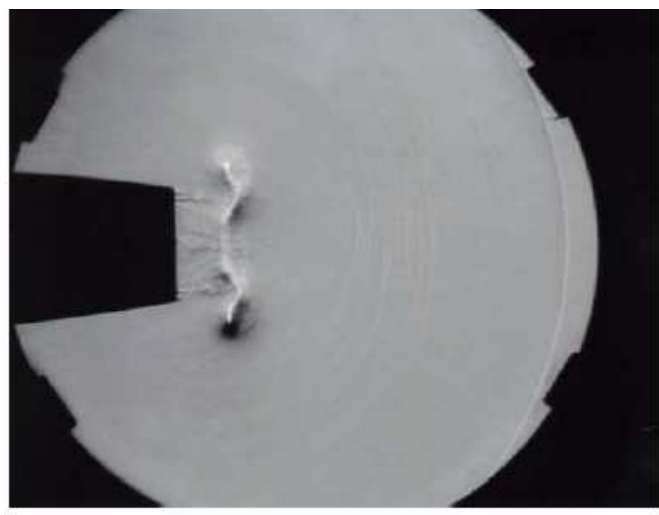

(a)

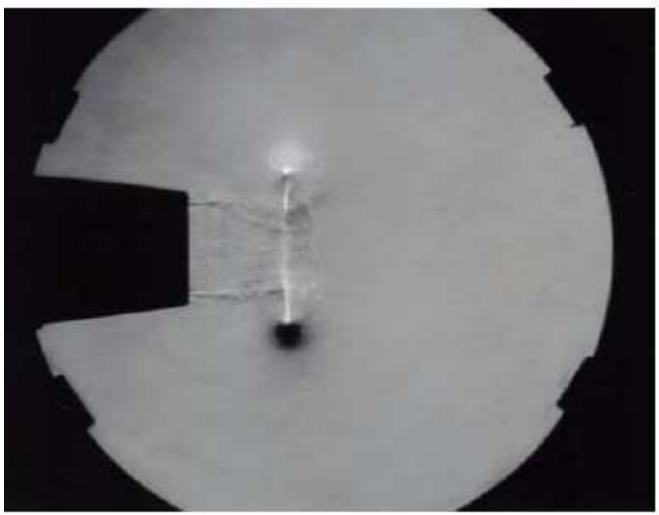

(c)

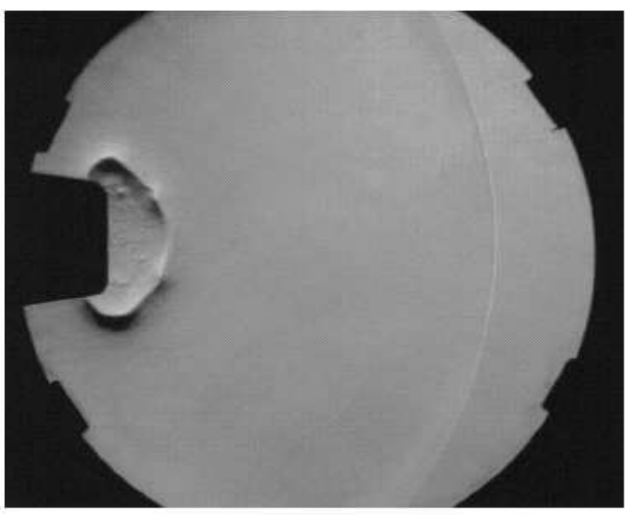

(b)

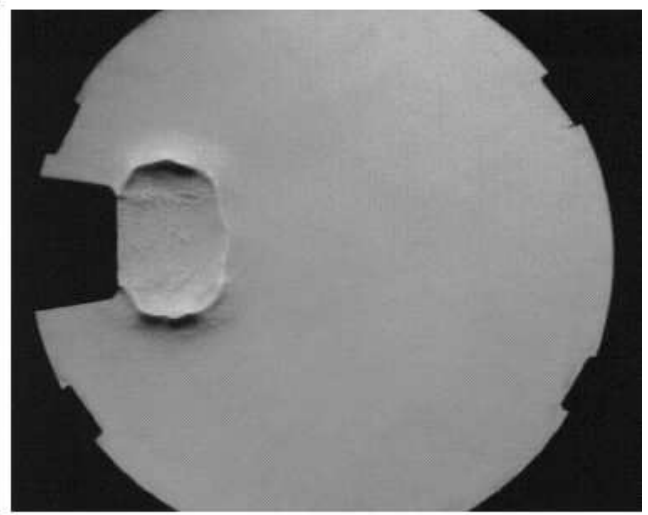

(d)

FIG. 22: Square nozzle, 4 bar, $t=$ (a) \& (b) $0.25 \mathrm{~ms}$, (c) \& (d) $0.45 \mathrm{~ms}$. ((a) and (c) are side views, (b) and (d) are oblique views) 


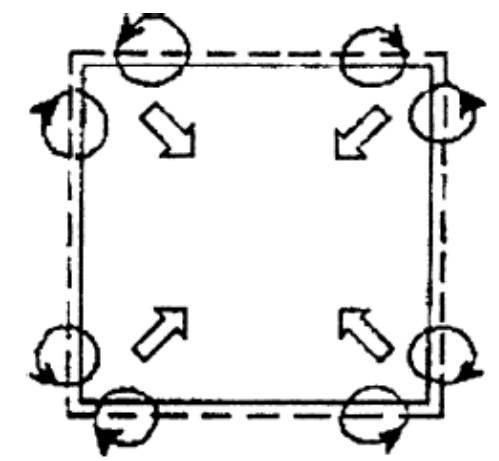

(a)

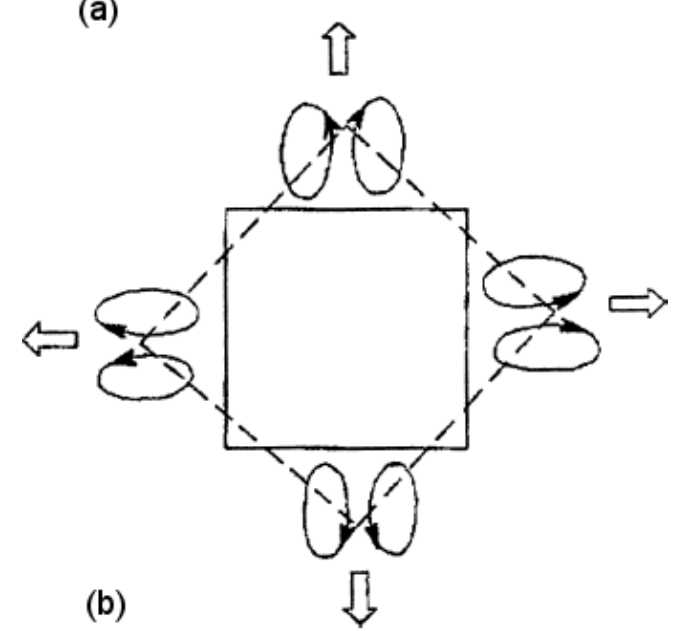

FIG. 23: Schematic of mean streamwise vorticity distributions in a square jet, (a) close to the jet exit, (b) further downstream. ${ }^{61}$

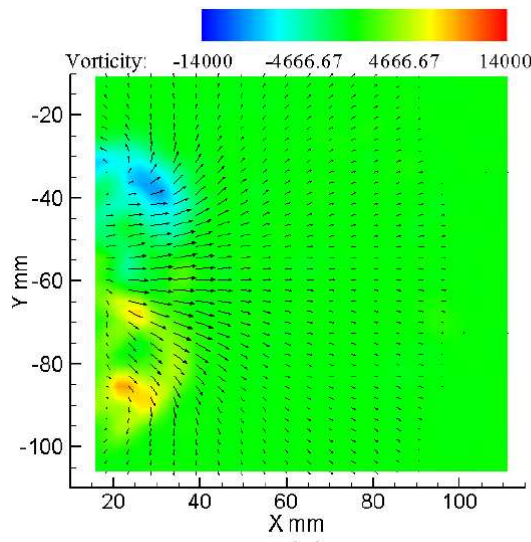

(a)

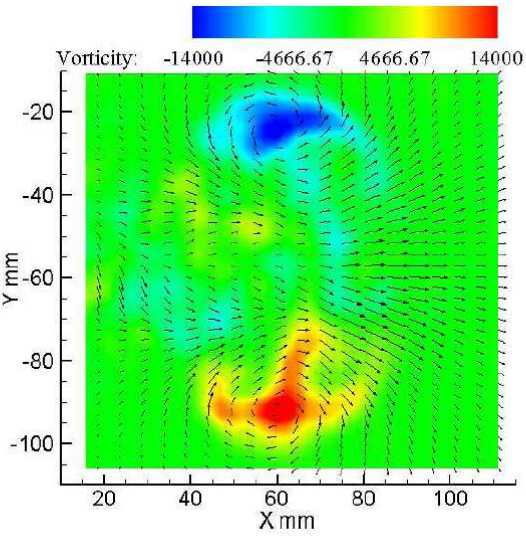

(b)

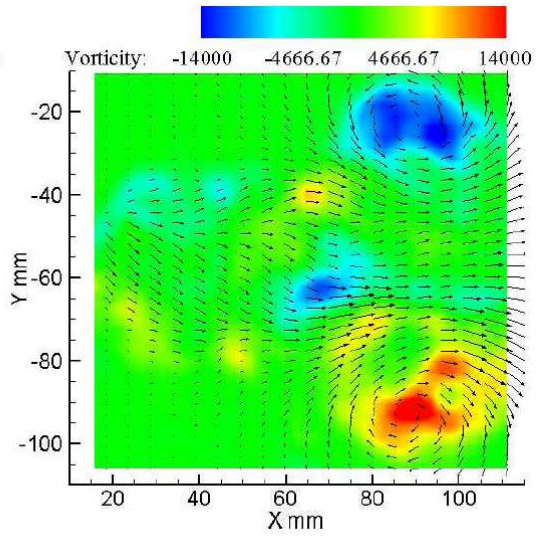

(c)

FIG. 24: PIV results for square nozzle, side view, $4 \mathrm{bar}, t=$ (a) $0.16 \mathrm{~ms}$, (b) $0.56 \mathrm{~ms}$, (c) $0.96 \mathrm{~ms}$. 


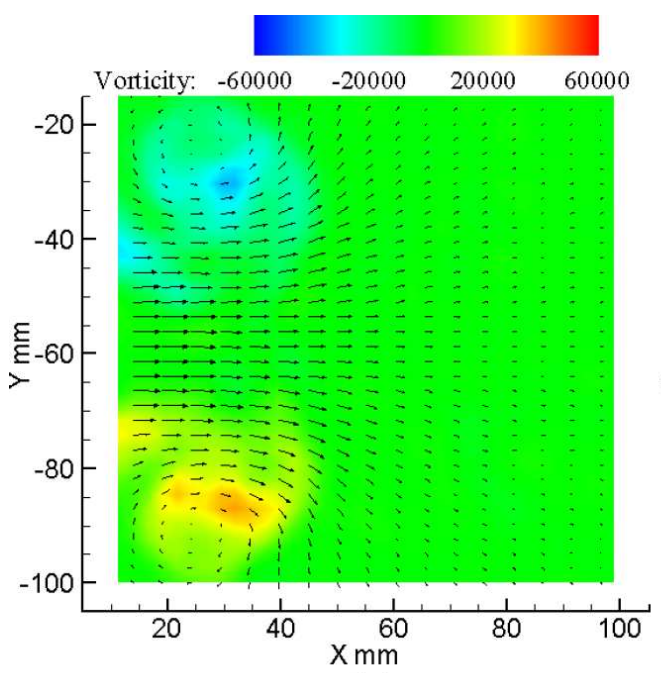

(a)

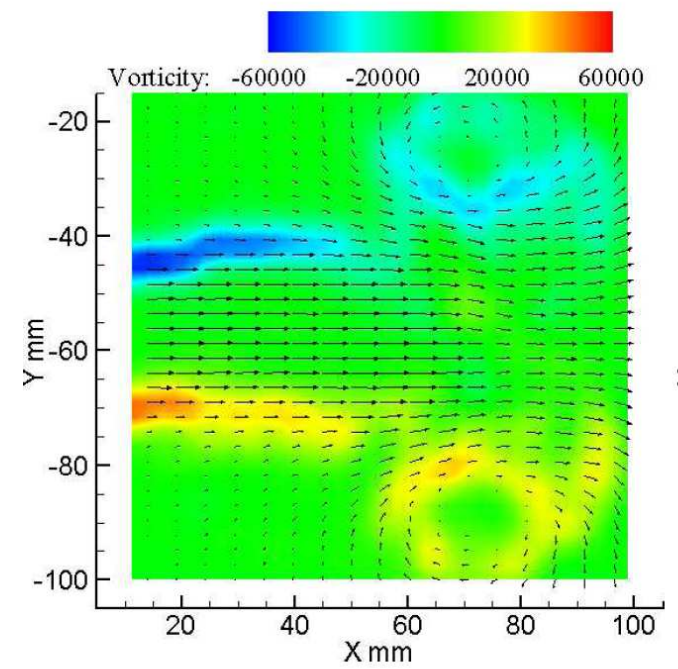

(c)

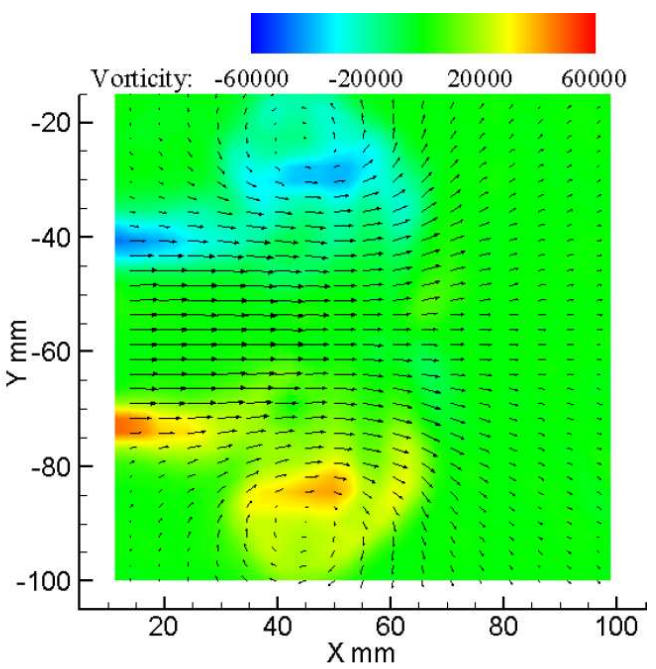

(b)

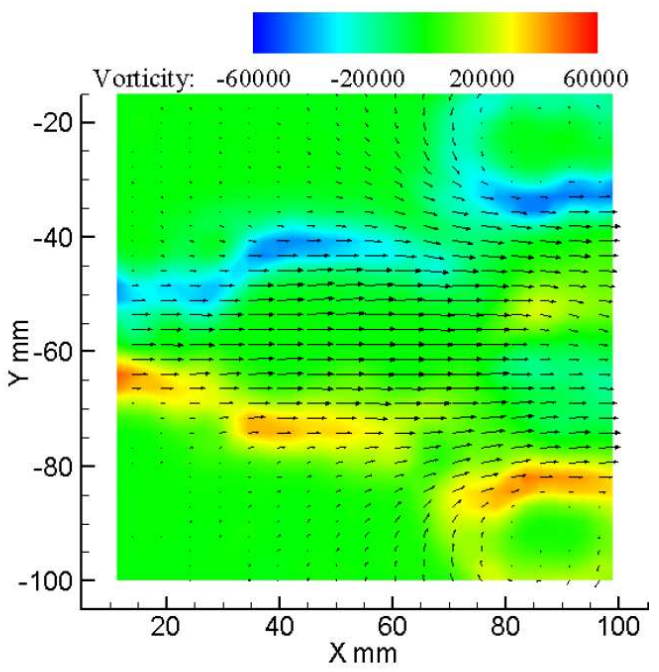

(d)

FIG. 25: PIV results for square nozzle, side view, $12 \mathrm{bar}, t=$ (a) $0.22 \mathrm{~ms}$, (b) $0.3 \mathrm{~ms}$, (c) $0.47 \mathrm{~ms}$, (d) $0.53 \mathrm{~ms}$. 


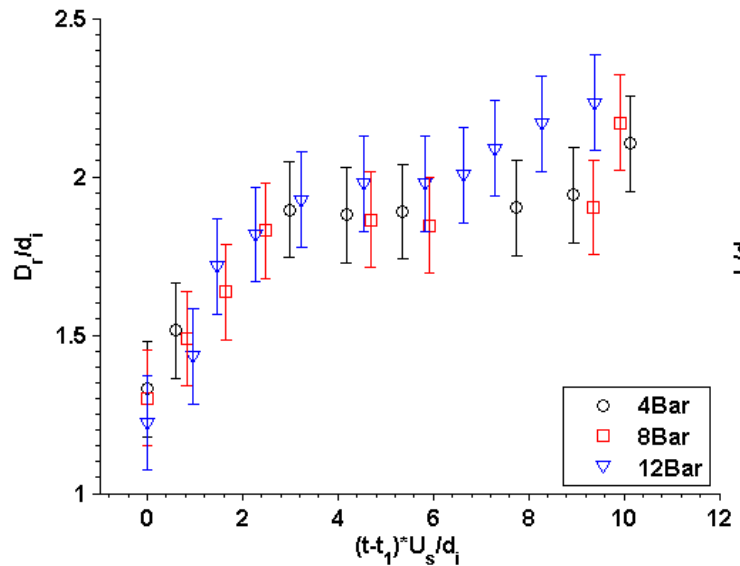

(a)

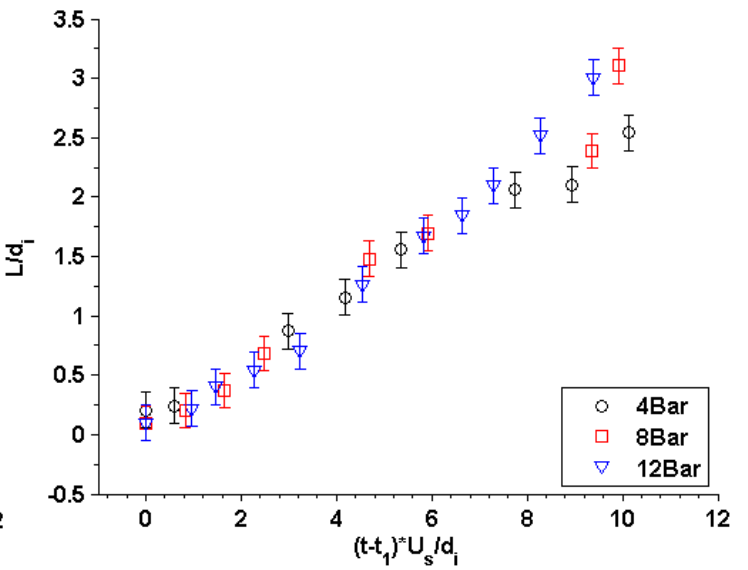

(b)

FIG. 26: Square nozzle variation of: (a) vortex loop diameter, (b) vortex loop position. 